University of Redlands

\title{
Film and Television Production Location Scouting - An Online GIS- Based Location Library
}

A Major Individual Project submitted in partial satisfaction of the requirements

for the degree of Master of Science in Geographic Information Systems

by

Mark E. Gilbert

Ruijin Ma, Ph.D., Committee Chair

Mark Kumler, Ph.D.

December 2016 
Film and Television Production Location Scouting - An Online GIS-Based Location Library

Copyright $@ 2016$

by

Mark E. Gilbert 
The report of Mark E. Gilbert is approved.
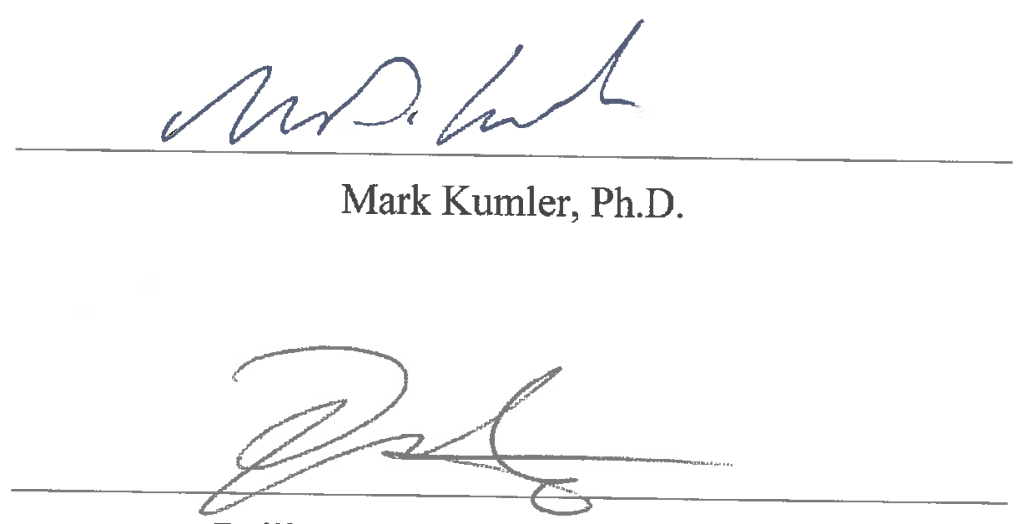

Ruijin Ma, Ph.D., Committee Chair

December 2016 



\section{Acknowledgements}

This project and my time at the University of Redlands would never have been possible without the help and support of many important people in my life. First, thank you to my wonderful husband, Don R. Cecil. Without his support and belief in me, I would never have submitted my admissions application in the first place. Don, you were always there with words of encouragement and support, to make edits to writing assignments and presentations, and to pull me back from the edge when things got a little out of control. You are a wise and successful man, and I strive to be more like you every day. This was a challenging year for us personally, but you were always able to take control of the chaos in our lives and keep me focused on my studies.

I also owe a debt of gratitude to my family (Rose, Sid, Scott, and Heather), who rallied around me in my time of need and insisted that I not give up. Your support was essential to my success. Thank you!

I want to give a big thankyou to my advisor and committee chair, Ruijin Ma, Ph.D. Your technical guidance during the project implementation was critical to my success. I greatly appreciate that you challenged me on my deadlines, and I am convinced this was the only reason I was able to finish on time. Also, I'd like to thank to my second reader and MS GIS faculty member, Mark Kumler, Ph.D. You introduced me to peanut butter pizza, and — on more than one occasion - the free Wi-Fi at Gourmet Pizza Shoppe prevented late assignments when the university network was down.

I'm grateful to my client Levi Vincent, executive director of the Greater Palm Springs Film Alliance. Working with you could not have been easier. You are a true professional, and you were always so understanding and accommodating as we worked through the pitfalls and learning experiences this project provided. Your interesting and engaging project idea reignited my love for classic Hollywood movies and introduced me to the world of filmmaking history. Another thankyou goes to University of Redlands alumnus and Cohort 14 member David Robinson, from the Coachella Valley Economic Partnership (CVEP), for bringing this project and for providing me with little doses of reality as someone who has been in this position before.

Last but certainly not least, thank you to Andrea Barrios, MS GIS project coordinator. You managed the chaos with a smile every day and made it look easy to keep multiple cohorts moving forward at the same time. From your gentle reminders about turning something in to your always having candy at your desk, your support was appreciated every day. It was such a quick year but I am looking forward to running into you on the Big Thunder Mountain Railroad. 



\begin{abstract}
Film and Television Production Location Scouting - An Online GIS-Based Location Library

by
\end{abstract}

Mark E. Gilbert

The film and television production industries are multibillion-dollar businesses for the State of California. When productions leave the state for other locations, the economic and workforce impacts on local communities relying on production business can be significant. The application of geographic information system (GIS) technology to the location scouting business is a novel approach to streamlining the location scouting process. Enhancing the site selection process by making it a web-based self-service endeavor, consolidating disparate data sources, and featuring local crew, talent, and filmfriendly businesses, the online location library assists the client in achieving key business efficiency objectives. The custom JavaScript online location library incorporates Esri, Inc. ArcGIS technology and simplifies the location scouting process for the client, makes it easier to find the right location, and highlights the Coachella Valley as an international production destination. This proof of concept web application demonstrates the use of web GIS technology that can be used by other local, regional, and state film offices. 



\section{Table of Contents}

Chapter 1 - Introduction ................................................................................................ 1

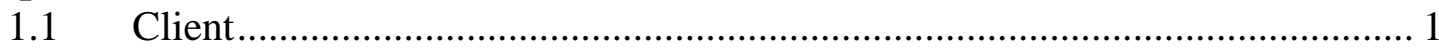

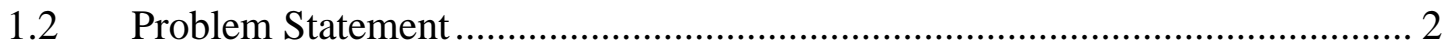

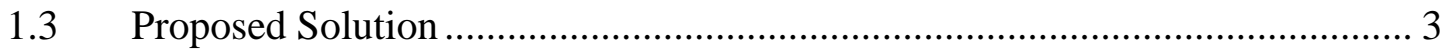

1.3.1 Goals and Objectives ............................................................................. 3

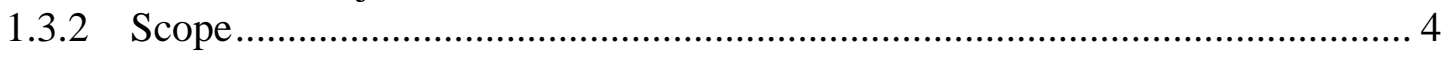

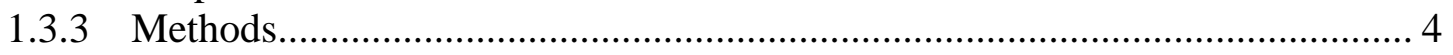

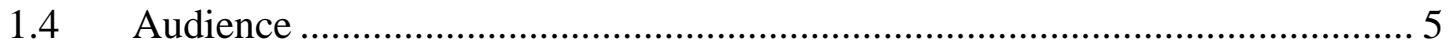

1.5 Overview of the Rest of This Report ........................................................ 5

Chapter 2 - Background and Literature Review ............................................................. 7

2.1 The Early Hollywood Studio System in California ........................................ 7

2.2 Runaway Productions - The How and the Why ……….................................. 8

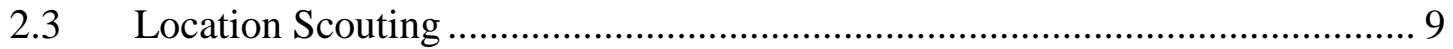

2.4 The Role of the Film Commission.............................................................. 10

2.4.1 The California Film Commission and CINEMASCOUT ................................ 10

2.4.2 The Greater Palm Springs Film Alliance ...................................................... 11

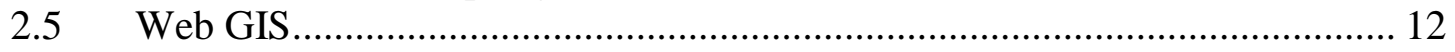

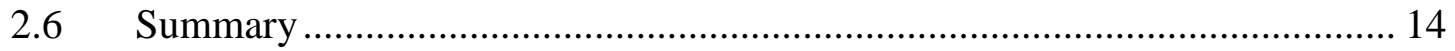

Chapter 3 - Systems Analysis and Design........................................................................... 15

$3.1 \quad$ Problem Statement ............................................................................. 15

3.2 Requirements Analysis …………………………................................. 15

3.2.1 Details of Functional Requirements............................................................. 15

3.2.2 Details of Nonfunctional Requirements....................................................... 17

3.3 System Design ................................................................................... 19

3.3.1 System interface requirements .................................................................. 20

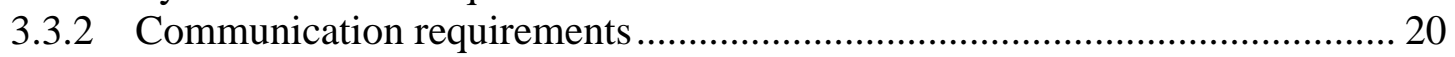

3.3.3 Hardware and software requirements ....................................................... 20

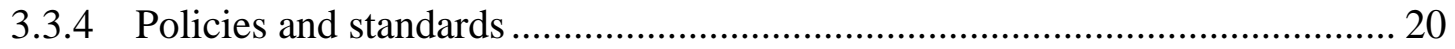

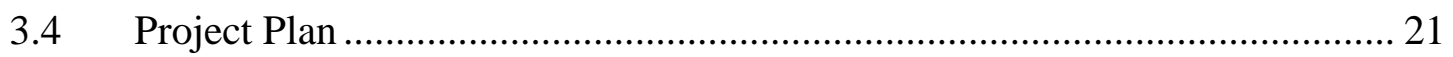

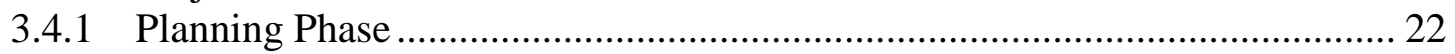

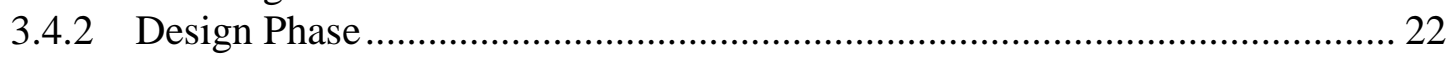

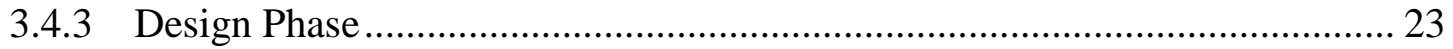

3.4.4 Implementation/Testing Phase ................................................................ 23

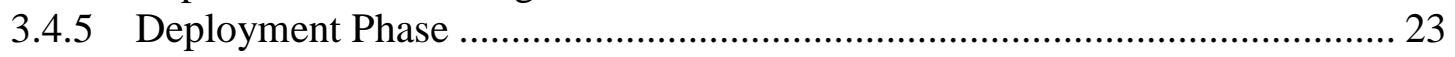

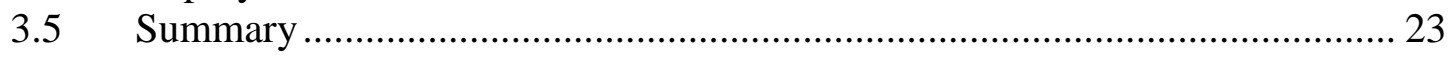

Chapter 4 - Database Design................................................................................................ 25

$4.1 \quad$ Conceptual Data Model ................................................................... 25

4.2 Logical Data Model .......................................................................... 26

4.2.1 Location Feature Class ............................................................................ 27

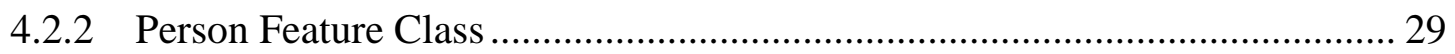

4.2.3 Vendor Feature Class ................................................................................. 29

4.2.4 Permit Data Feature Class......................................................................... 30 
4.2.5 Supporting Nonspatial Tables and Relationship Classes................................ 31

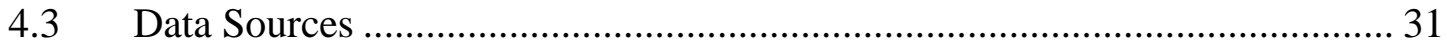

4.4 Data Collection Methods ..................................................................... 31

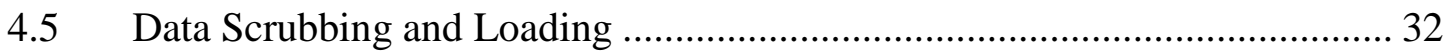

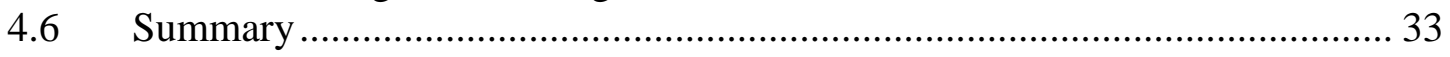

Chapter 5 - Implementation................................................................................................. 35

$5.1 \quad$ Publishing the Hosted Feature Layer ................................................................. 35

5.2 Developing the Web Application...................................................................... 35

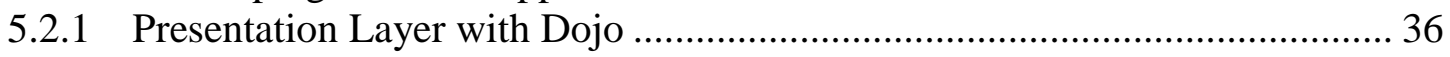

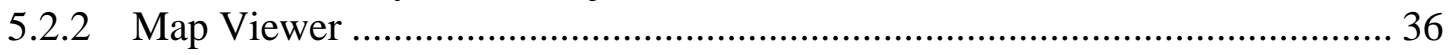

5.2.3 Symbolizing Feature Services and Pop-ups................................................... 39

5.2.4 Content Pane and Filter Feature.................................................................... 41

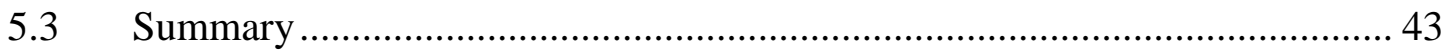

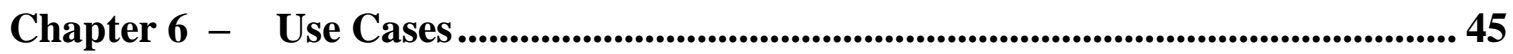

6.1 Use Case: Location Scouts...................................................................... 45

6.2 Use Case: Partner Film Commission Members …………………………...... 50

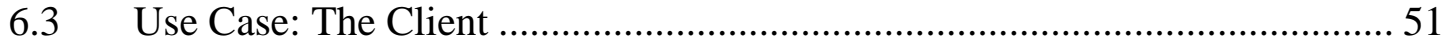

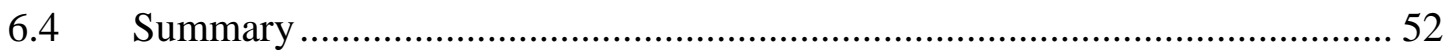

Chapter 7 - Conclusions and Future Work ................................................................... 53

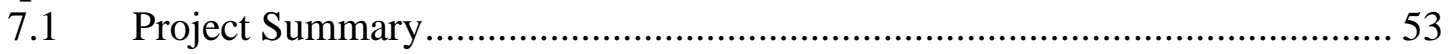

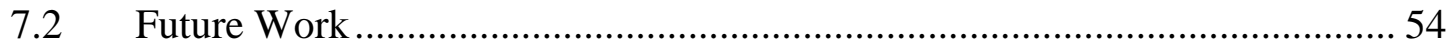

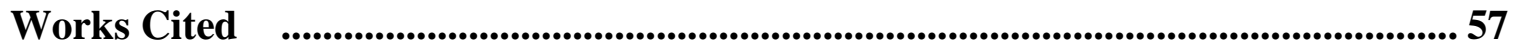

Appendix A. HTML Source Code ........................................................................................ 59

Appendix B. JavaScript Source Code ................................................................................. 61

Appendix C. CSS Source Code.......................................................................................... 71

Appendix D. Python Script ….............................................................................................. 73 


\section{Table of Figures}

Figure 1-1: Cities of the Coachella Valley Making Up the Project Study Area .......... 2

Figure 2-1: CINEMASCOUT at the California Film Commission Website............. 11

Figure 3-1: Graphic representation of application components............................. 19

Figure 4-1: Location library conceptual data model ............................................26

Figure 4-2: Geodatabase design indicating feature and relationship classes ............27

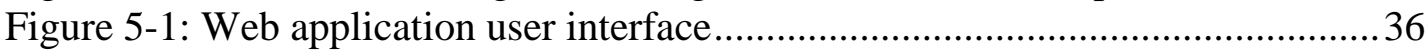

Figure 5-2: Center region of the web application user interface ............................ 37

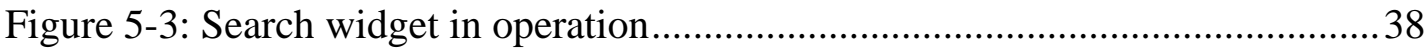

Figure 5-4: Components of the user interface working together ..............................39

Figure 5-5: All locations in the library display by default .......................................40

Figure 5-6: Left region of the user interface showing the location filter feature....... 41

Figure 5-7: Location filter feature and ContentPane creation workflow .................. 42

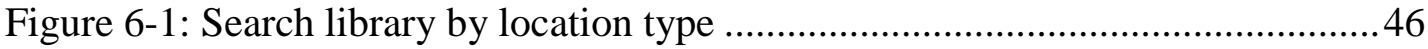

Figure 6-2: Location pop-up displaying relevant site and production information ... 47

Figure 6-3: Application interface showing local business information .....................48

Figure 6-4: Location filter feature showing only one category ...............................49

Figure 6-5: Film permit details for Riverside County .............................................50

Figure 6-6: Administrative interface for adding locations...................................52 



\section{List of Tables}

Table 1. Online Location Library Functional Requirements .................................. 15

Table 2. Online Location Library Nonfunctional Requirements .............................. 18

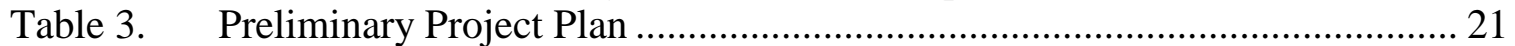

Table 4. Location Feature Class Key Attributes ....................................................... 28

Table 5. Person Feature Class Key Attributes ....................................................... 29

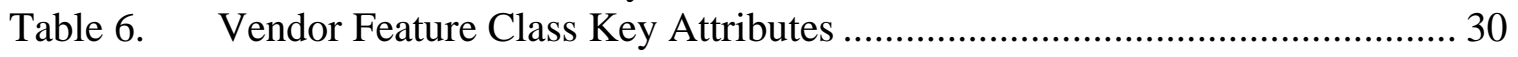

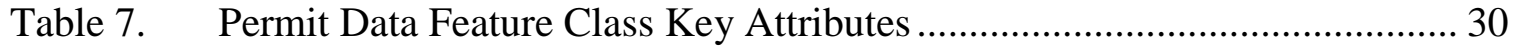

Table 8. Example of Vendor with Multiple Categories ............................................ 33 



\section{List of Acronyms and Definitions}

$\begin{array}{ll}\text { API } & \text { Application Programming Interface } \\ \text { CFC } & \text { California Film Commission } \\ \text { CVEP } & \text { Coachella Valley Economic Partnership } \\ \text { GIS } & \text { Geographic Information System } \\ \text { GPSFA } & \text { Greater Palm Springs Film Alliance } \\ \text { MIP } & \text { Major Individual Project } \\ \text { REST } & \text { Representational State Transfer } \\ \text { UI } & \text { User Interface } \\ \text { PMI } & \text { Project Management Institute } \\ \text { CCS } & \text { Cascading Style Sheet } \\ \text { HTML } & \text { Hypertext Markup Language } \\ \text { IP } & \text { Internet Protocol } \\ \text { IDE } & \text { Integrated Development Environment } \\ \text { AGO } & \text { ArcGIS Online } \\ \text { NAD } & \text { North American Datum } \\ \text { SP } & \text { State Plane } \\ \text { CSV } & \text { Comma Separated Values } \\ \text { VBA } & \text { Visual Basic for Applications } \\ \text { WGS } & \text { World Geodetic Survey } \\ \text { URL } & \text { Uniform Resource Locator } \\ \text { QA/QC } & \text { Quality Assurance / Quality Control }\end{array}$





\section{Chapter 1 - Introduction}

Film, television, and media production are major industries in California, generating significant economic impact through expenditures on wages, goods and services, sales, and use taxes. However, every year, production projects leave California for other states and countries offering significant incentives intended to attract the jobs and economic benefits these projects bring to local and regional economies. In order to help mitigate these losses, in 1985 California governor George Deukmejian established the California Film Commission (CFC) (Hozic, 2001). This action set the stage for the subsequent creation of more than 60 local and regional film commissions to develop solutions and coordinate stakeholders to attract and retain production projects in California (California Film Commission, n.d.). The client proposes using geographic information system (GIS) technology to improve the efficiency of location scouting and finding local crew, talent, and film-friendly businesses. This report presents a map-based online location library solution that serves as a one-stop resource for location scouting.

\subsection{Client}

The project client was the Greater Palm Springs Film Alliance (GPSFA), led by executive director Levi Vincent. The mission of this public-private partnership of film, television, video, and affiliated professionals was to promote the Coachella Valley as a domestic and international production destination and support locally based crew, talent, and film-friendly businesses (Greater Palm Springs Film Alliance, n.d.).

Palm Springs, California is just one of several desert cities, approximately 124 miles east of Los Angeles, which make up the Coachella Valley. The client brought more than eight years of experience and industry knowledge to this project and had a successful record of promoting the Coachella Valley as a desirable production location. The GPSFA's business objectives include featuring local crew, talent, and film-friendly businesses in order to drive economic activity and to advocate for the Coachella Valley as an ideal location for production activity. With the support of the Coachella Valley Economic Partnership (CVEP), GPSFA works to promote a year-round economy and stimulates job creation in the film and television production industries (Coachella Valley Economic Partnership, n.d.).

The role of the client in this project included two important responsibilities: defining the business requirements and reviewing and approving all web application functionality. Moreover, the client provided all photos and datasets for locations, people, and local businesses. The client was also responsible for ongoing maintenance that would include editing location information, updating location attributes, and adding or deleting site information.

The client did not have the GIS infrastructure necessary to provide long-term storage of the data and GIS artifacts. Therefore, all development and testing of the product were performed on internal equipment using university GIS infrastructure. The final web application was migrated to the client's corporate web server, and the university resources will continue to provide back-end GIS infrastructure for one year after the completion of this project. 


\subsection{Problem Statement}

The client's manual process for identifying appropriate production locations within the Coachella Valley was time-consuming. The location scouting process often required that the client make simultaneous inquiries to multiple local and regional film commission offices. Due to the client's slow process and tight turnaround times for productions, potential projects were going to other states and countries, resulting in lost economic gain. The U.S. Department of Commerce defines these as runaway productions, which result in lost revenue and jobs for California. This has been an increasing trend since the late 1990s (U.S. Department of Commerce, 2000). Figure 1-1 maps the Coachella Valley in relation to the rest of the state and highlights the cities making up the project study area.

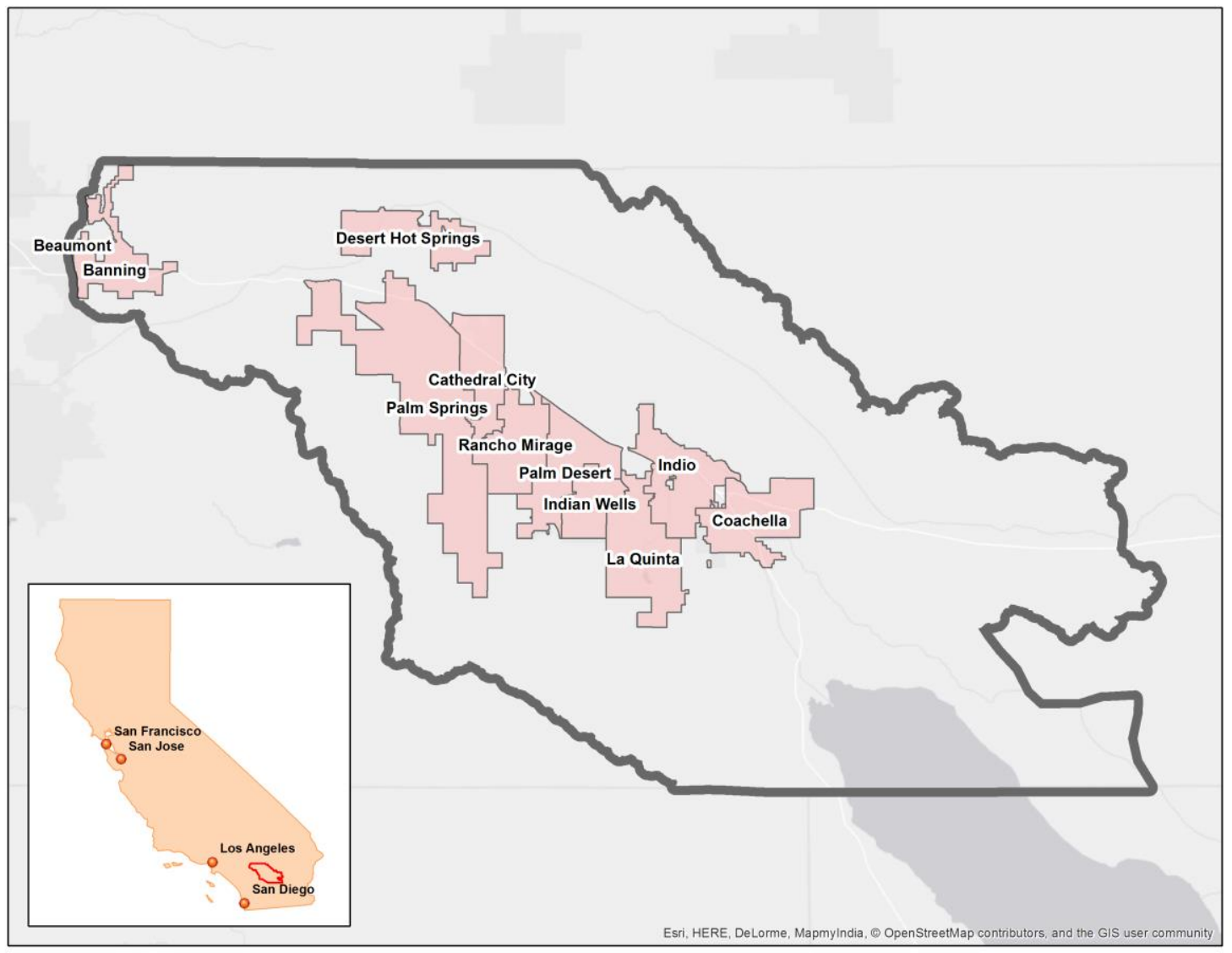

Figure 1-1: Cities of the Coachella Valley Making Up the Project Study Area

Film and television production resource and location scouting are spatial endeavors. Thematic neighborhoods, iconic locations, and scenic views all share spatial qualities and can be effectively searched and reviewed on a map in context with their location and relationship to one another. Every jurisdiction (local government, tribal organization, and federal agency) has its own permitting requirements, fees, and procedures for filming approval. The process of identifying locations of interest can be time-consuming, and determining which agency, organization, or private party has jurisdiction over a location 
can be a challenge for those unfamiliar with the area. These factors can force even the most patient producer to lose interest and go elsewhere.

Another challenge is finding local talent and film-friendly businesses available for hire in the vicinity of a desired site (Vincent, 2016). Bringing these components together into a one-stop search tool with an intuitive interface would set the client and the Coachella Valley apart as regional leaders in the production resource and location scouting industry. Successfully booking more productions will stimulate economic activity and job growth for cities in the Coachella Valley and serve as a model for other regional film commissions throughout the state.

The previous process of location and resource scouting was manual and had limited online tools covering regional locations like the Coachella Valley. When a potential client contacted the GPSFA office, staff would gather requirements to determine suitable locations meeting the production's needs. The GPSFA would present potential locations to the scout, along with details about site ownership, filming-related restrictions, jurisdictional authority, and state or local permit and fee requirements. If the production also sought crew, equipment, or other professional referrals to support the production, GPSFA staff would research additional databases for the requested services.

\subsection{Proposed Solution}

The online location library needed to use modern web technology to streamline the location scouting process so that staff could refer producers and scouts to the GPSFA self-service website and focus precious resources on follow-up inquiries. This process would meet the client's goal of retaining film and television productions and benefitting the local economy. The client needed an interactive online tool in order to search and identify potential production locations throughout the region. The spatially enabled tool would need to deliver location information along with details about the site, jurisdictional authority, permit requirements, and fee information. It also had to provide information about local crew, vendors, and other film-friendly businesses, thus streamlining the scouting process by providing a one-stop resource for production scouts.

Location scouts and producers search the site database using keywords or phrases to find locations matching their criteria. The tool provides functionality for selecting from prepopulated lists of site categories and displays those locations on the map. After a user executes a search, sites matching the criteria will appear on a map with interactive informational pop-up windows including the site's agency jurisdiction, contact information, fees and permit requirements, and public safety and/or insurance conditions as well as incentives offered by the owner. Standard map interface features would include functions like zooming in/out, panning, and returning home. Locations for crew, vendors, and businesses would be displayed on the map and have their own pop-up windows for additional details.

\subsubsection{Goals and Objectives}

The goals of this project were to deliver a spatially enabled database with online, mapbased location and vendor search tools that would streamline the scouting process, enhance the efficiency of finding suitable locations throughout the Coachella Valley, and increase the number of productions booked by the client. 
The typical usage scenario of the location library allows the user to search for locations using multiple methods and presents detailed information about the site on a map. Online search tools make the client's job easier by giving location scouts and producers a self-service model to facilitate and encourage production in the Coachella Valley.

The Greater Palm Springs Film Alliance business objectives include highlighting the Coachella Valley as an attractive production destination. The online location library supports this objective by improving the location scouting process and making it easier to hire local professionals. All the information needed by producers and scouts is available in one place and comes from multiple city, county, federal, and tribal jurisdictions. Providing a self-service tool enables many scouts and producers to find what they need in a matter of hours rather than days and without requiring the assistance of GPSFA staff, who, in turn, are free to focus on requests that are more complex. This tool could also remove barriers to securing project approval. If the GPSFA consequently doubles the average number of annual productions booking Coachella Valley locations and local professionals, then the organization's overall business objective of highlighting the region and stimulating year-round job creation will be met.

\subsubsection{Scope}

The online location library is a web-based application that allows a user to search for production locations and local crew, talent, and businesses by using keywords or phrases. Multiple search methods allow flexibility in locating in-depth information about a site's characteristics and other production-related details.

Search methods available include full text, visual exploration on a map, and interactive filtering via categories. The location library uses standard map interface functionality for zooming in/out, panning, centering, and layer selection. Search, Query, and NearMe widgets provide functionality for attribute and proximity searches.

Results of search and filtering actions are displayed on the map and provide the user with detailed production-related information in pop-up windows and text boxes within the map display. Production-related information includes the jurisdictional authority, details about permitting, fees, insurance and public safety requirements, and available incentives. The web search tool provides the user with service and contact information for local crew, talent, and film-friendly businesses in the vicinity of the desired locations. Category definitions for locations, services, and businesses were provided as part of the data delivered by the client.

\subsubsection{Methods}

The process for project implementation involved planning the work to be performed and documenting a timeline. This process included a preliminary plan for the methods to be used in delivering the client's web application. These methods included data review, data cleaning, geocoding, geodatabase development, ArcGIS Online processing and configuration, and JavaScript web development. The associated activities, tasks, technologies, and techniques for each phase are outlined in later sections.

The project began with a data collection process. The client provided data in

spreadsheet format and included location, person, and local business records. The project 
team reviewed these data for accuracy and compliance with the agreed-upon data scheme for each record type, made corrections where necessary, and prepared the data for import into Esri's ArcGIS for Desktop software.

The project utilized ArcGIS for Desktop software to develop and test the geodatabase and publish the feature classes in ArcGIS Online as a hosted feature service. The geodatabase employed domains and subtypes to ensure that users and administrators type correct and accurate data via the web application.

Once the geodatabase was completed, the feature classes were published in ArcGIS Online as Representational State Transfer (REST) services. ArcGIS Online web mapping tools, standard JavaScript web development techniques, and Esri ArcGIS API for JavaScript were used to build the custom web mapping application.

\subsection{Audience}

The project and report were prepared for the Greater Palm Springs Film Alliance, but it has relevance for other film and television professionals. The GPSFA's interest is efficient promotion of the Coachella Valley and attracting location scouts, producers, directors, and other film commissioners to the area. Nontechnical readers of this report would include film and television industry professionals seeking information about online location library tools. Technical readers would include web programmers and GIS professionals interested in application development for cataloging, storing, and retrieving site information within a mapping context. This report assumes readers will have at least some exposure to web development, web programming, and GIS techniques.

\subsection{Overview of the Rest of This Report}

The remainder of this report covers specific topics including detailed design, implementation, issues, and possible future work. Chapter 2 provides background details about the emergence of location scouting, citing the political, social, and economic events that drove its evolution. A review of the literature about runaway productions and location scouting provides context and rationale for web-based location scouting tools and film commissions, and includes what the State of California has done to provide incentives and retain productions. Chapter 3 examines the components that made up the preliminary design processes, system analysis, design, and project planning. The initial system analysis included functional and nonfunctional requirements gathering with the client, as well as preliminary design and project timeline development. This chapter also includes the project plan. Chapter 4 covers the actual design work performed in support of the final product. Beginning with conceptual database design, the chapter moves on to data collection and cleaning, quality control steps taken to ensure that the data were accurate, and how the prepared data were published online. Chapter 5 details the online development portion of the project and includes specifics on how JavaScript, the Dojo toolkit, and ArcGIS API for JavaScript were used to design and test the business logic and presentation layers for the online location library. Chapters 6 reviews the results of the final product, and the report wraps up with concluding remarks and possible areas of future improvement in chapter 7 . 



\section{Chapter 2 - Background and Literature Review}

Chapter 2 introduces a brief history of filmmaking in Southern California as context for why the industry continues to be essential to the local economy. In addition, a working definition will be established for the term "runaway production" as well as an explanation of why it is worthy of discussion. These topics lay the groundwork for understanding the negative economic impacts runaway productions have had on the California economy and why elected officials at various levels of government have intervened. With this brief history as a backdrop, sections 2.3 and 2.4 explain the roles film commissions play in stemming the exodus of productions to other states and countries. Finally, the chapter closes by making the case for why California film commissions have succeeded at reducing runaway productions for California.

\subsection{The Early Hollywood Studio System in California}

It is commonly believed that Hollywood, California, was as the birthplace of the modern film and television industry, dating back to the early 1900s. The first permanent film studio was built in Los Angeles in 1909 (Hollywood Film Office, 2008). Today, these industries generate a $\$ 30$ billion a year in business for California and create an estimated 160,000 jobs (Southern California Association of Governments, 2014). Film and television are synonymous with California's heritage, brand, and reputation. To bolster these attributes, film commissions and politicians throughout the state compete to promote their local and regional areas as premier destinations to base film, television, and other media productions. Commissions compete for the direct economic benefits of these productions but collectively work toward the common goal of keeping production activity in California.

As movie studios grew in size and power during the 1920s, most movie stars were under direct contract with a studio. This meant that movie studios carefully staged and choreographed all aspects of an actor's professional and personal life to promote and maintain "star" status. This period of mass production gave studios great control over their actors, on and off the screen. The studio system era also gave movie producers control over the information the public received about their movies. However, as productions began to leave Los Angeles, the studios not only lost control of the stars but also the publicity information that was released about a movie production (Hozic, 2001).

Authors who write about Hollywood, like Aida Hozic, characterized this period in early Hollywood history as localized movie production. Every aspect of a film was shot entirely on the studio's soundstage or at its movie ranch. Within the four walls of a stage, places as distinctive as ancient Rome, Brooklyn, or the American South were re-created with skill and precision by the Hollywood filmmaker. Keeping costs down may have been the primary reason for the strictly controlled environment of a soundstage, but it was also a way movie producers exerted their control over production (Hozic, 2001).

Following World War II, Hollywood's complete control over movie production weakened. The conditions that had that allowed the studios to control their stars and movies rapidly disappeared, and a new era of geographically dispersed moviemaking emerged. 


\subsection{Runaway Productions - The How and the Why}

The literature reviewed for this report clearly documents the negative economic impact of productions moving out of the state and the country and includes lost jobs and revenue decline for public agencies, tribal organizations, and companies of all sizes that support the industry. A film or television production that is conceived of locally but is produced elsewhere is called a runaway production. This issue has been of major concern to the industry - so much so that federal, state, and local governments have enacted tax laws and streamlined permit requirements to attract and retain production activities (Battista, 2014). What follows is a discussion of what runaway productions are, the historical context for how they became a problem, and what lawmakers and politicians are doing about them.

During World War II, political and economic circumstances forced many European nations to shut down movie theaters, and U.S. film exports came to a standstill. By the end of the war, U.S. movie studios had amassed a stockpile of unreleased films and were eager to return to the lucrative business of exporting films to Europe. Cash-strapped countries like England were doing all they could to prevent a currency shortage, enacting efforts including excessive tax on imports of American-made films. Growing labor issues in Hollywood, combined with strict British import tariffs and revenue caps imposed on filmmakers, proved to be challenging but not impossible for U.S. movie producers to overcome (Yale, 2010). Great Britain and the United States came to an agreement called the Anglo-American Film Pact, which allowed British theaters to resume showing American-made films and eliminated the $75 \%$ tax on foreign film revenues in Britain. Under this agreement, U.S. movie producers were able to reduce their tax bill but still had to operate under policies that put a cap on revenue that could be taken out of the country (“Anglo-American Film Deadlock Broken by Pact," 1948).

Movie producers were also able to access some of the capped revenue, thanks to a generous provision of the British government that allowed them to invest revenues above the cap back into the foreign economy - specifically, into film production. A byproduct of the Anglo-American Film Pact resulted in American dollars financing foreign films and paying wages to non-American workers. This perceived negative impact on American film industry workers did not sit well with Hollywood union leaders. The resultant labor conflict only intensified the growing turmoil between Hollywood labor unions and movie producers (Johnson-Yale, 2015).

Hollywood labor unions' increasing power in the late 1940s and early1950s greatly influenced the growth of runaway productions. Organized labor in the United States was becoming increasingly expensive as tradespeople became more specialized and union leaders began demanding better pensions and benefits for their members. Movie producers quickly learned that they could reduce production costs by shipping projects overseas where they were already funding production services and labor.

Authors agree that political, economic, and social issues occurring after World War II led to much discussion about the perception of runaway productions. In her dissertation on the history of Hollywood outsourcing, Camille K. Yale detailed political and economic conditions that existed in the mid- to late 1940s that resulted in a rise in runaway productions (Yale, 2010). She provided historical context for this rise and the increasing fear of Hollywood labor unions that studios and film producers were sending 
jobs overseas (Johnson-Yale, 2015). Hozic, in her book titled Hollyworld, concurred that labor and political conditions led to more runaway productions, but according to Yale, labor and media used the term runaway production almost exclusively to raise fears rather than explain a phenomenon (Hozic, 2001). The Hollywood industry may have originally coined the term to mobilize support for a political cause. The term, however, remains in common parlance today. As recently as 2014, Paul Battista continued to use the term and attached far more weight to its effects by stating that runway productions are not simply a labor or international trade issue but, rather, a U.S. tax code issue that "indicates the need for an active response by the U.S. government" (Battista, 2014, pg 304).

Over the past 10 years, runaway productions have negatively affected California's economy because of lost jobs and revenue. According to Kevin Klowden and Anusuya Chatterjee (2010), in 2008 the film industry generated $\$ 25$ billion and created 167,000 jobs in the state, based on data from the California Employment Development Department (Klowden, Chatterjee, \& Hynek, 2010).

These results came on the heels of a peak of $\$ 21$ billion in 1997, followed by a significant drop in 1998 and a recovery in 2004 with \$24 billion in output. Jobs numbers in the late 1990s follow a similar downward trend that "coincides with a push by Canada, the United Kingdom, and Eastern Europe to build production facilities and cultivate local talent" (Klowden et al., 2010).

According to the California Film Commission (CFC), between 2003 and 2008 California experienced a $50 \%$ decrease in the number of studio feature films (Business Transportation and Housing Agency, 2009). The revenue and jobs decline experienced by the California film industry over this period indicated an exodus of productions from the state; simultaneously, there was a well-documented growth of production and talent employment in Canada and overseas. According to Kevin Klowden, Anusuya Chatterjee, and Candice Flor Hynek (2010), industry output has remained relatively flat since 2004, and jobs numbers have even dipped below the high in the same year.

The California State Legislature finally took action in 2009 and introduced the California Film and Television Incentive Program in an attempt to address the issue of productions leaving California. This state program involved a series of tax incentives designed to keep film and television productions in California. With over 40 other states competing against California through lucrative tax credits to lure productions, California was trying to retain the production activity that remained.

\subsection{Location Scouting}

Location scouting is the term used by industry professionals to describe the process of finding a film or television production location outside a studio sound stage. A director envisions the aesthetic for a scene, and a location scout finds the places that bring that vision to life. Re-creating large, dangerous, or historic locations inside a sound stage can be prohibitively expensive, unrealistic, or even unsafe. When there is a need to bring a more authentic look and feel into a scene, a location production is preferred over sets on a soundstage. It is the responsibility of the location scout to understand the filmmaker's requirements and use all available tools and resources to find the appropriate locations. 


\subsection{The Role of the Film Commission}

A film commission is typically a nonprofit organization established by public or private entities to coordinate between local government and film, television, and media productions. These commissions assist industry professionals with identifying locations; navigating the film permit approval process; and, often, finding local businesses that provide support services. Early film commissions originated in the well-established movie and television production cities of Los Angeles and New York. According to Marco Cucco and Giuseppe Richeri (2011), "film Commissions were thus the response to the growing need for film companies to have a local government liaison able to provide and coordinate public and private services for film shoots" (Cucco and Richeri, 2011, p. 4). Film commissions are established to attract all kinds of productions by "promoting the environmental characteristics of its region and providing a series of free services aimed at facilitating audiovisual production in its region" (p. 8). Film commissions developed around the world as the popularity of location filming grew. Surprisingly, it was not until 1985 that California set up its first statewide commission called the California Film Commission.

\subsubsection{The California Film Commission and CINEMASCOUT}

In 1985, California governor George Deukmejian signed into existence the California Film Commission (CFC). While the CFC alone did not turn the tide of runaway productions leaving California, it did establish a statewide one-stop shop for movie producers, providing location scouting and other support services envisioned to make production in California easier and more enticing.

One service offered by the CFC is CINEMASCOUT, an online location library claiming to hold data on thousands of searchable locations throughout the state.

CINEMASCOUT is searchable by keyword, geographic area, style, and other methods. Figure 2-1 shows the CFC CINEMASCOUT main interface. 


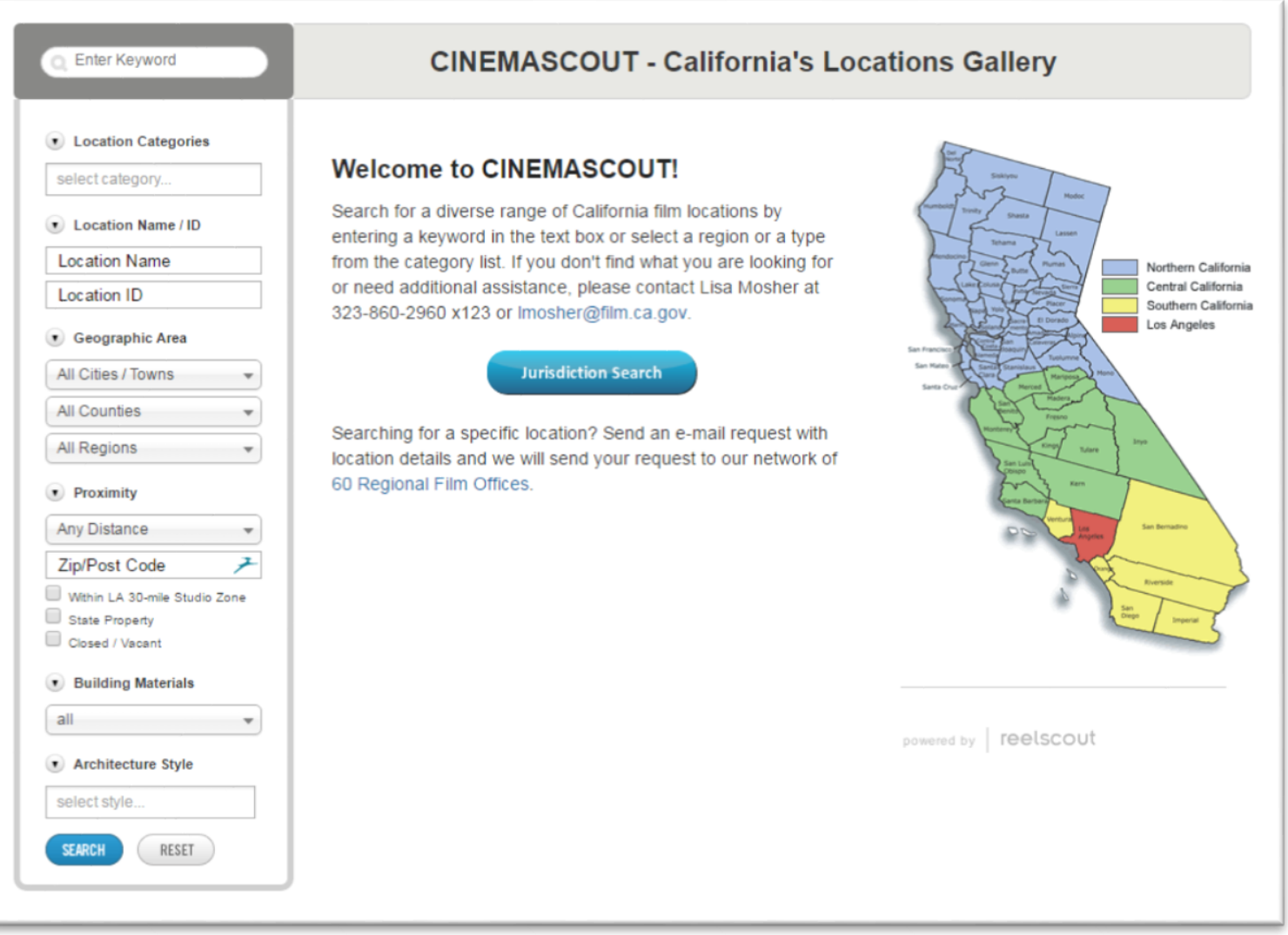

Figure 2-1: CINEMASCOUT at the California Film Commission Website (Reprinted from the California Film Commission website CinemaScout page. Retrieved on October 27, 2016, from http://ca.reelscout.com/loc.aspx. Public domain.)

CinemaScout appears to be an effective solution. However, according to some regional film offices, it is outdated, does not offer a user friendly experience, contains limited mapping capability, and is not easy to use on mobile devices (Vincent, 2016). CinemaScout also lacks the ability for a movie producer to identify local film-friendly businesses in proximity to a location. A production must factor in these local support services as part of the overall budget. Termed "below-the-line" costs, they are associated with nonartistic or noncreative crew and can be some of the most costly in a production (Cucco \& Richeri, 2011). Identifying and using nearby businesses, rather than shipping everything to a production site, helps keep location shooting costs under control. While statewide film commissions provide valuable services to the larger production community, the regional and local commissions bring expert knowledge about locations and business resources critical to location production.

\subsubsection{The Greater Palm Springs Film Alliance}

Since 2014, the Greater Palm Springs Film Alliance (GPSFA) has worked to redefine how large and small productions are attracted to the region. Armed with a new business plan, GPSFA executive director Levi Vincent uses the online location library as a twenty- 
first century tool to make his job easier while attracting an increasing number of productions to Palm Springs (Vincent, 2016). The GPSFA is at a critical juncture and understands that, in order to be competitive, the cities that make up the Coachella Valley must offer more than just tax incentives and permit support. If the GPSFA does not take advantage of current mapping and web technologies to improve the regional location scouting process, problems associated with runaway productions like lost revenue and jobs will continue to get worse.

Unlike statewide film commissions, regional and local film offices offer unique benefits. These smaller film commissions are often run by local experts who bring firsthand experience with the surrounding communities, locations, and businesses. A smaller film office also can provide personal contacts with individual and organizational owners of particular production sites. This is valuable because personal relationships often influence how quickly or easily a site gets booked.

The result of this project puts the online location library into the hands of the resident film office, helping to ensure the location inventory is complete and up-to-date with the latest details, pictures, and contact information. Additionally, the process of growing the online location library captures the area's institutional knowledge that might otherwise be lost.

The Coachella Valley and the city of Palm Springs, both closely associated with Hollywood, were the focus area for this project. The Coachella Valley includes nine distinct cities, each with its own character and charm. Throughout its history, the area has attracted U.S. presidents, celebrities of all genres, and tourists and adventure seekers. During the 1920s and the era of the studio system, actors were under tight control, required to stay within two hour's distance of the movie studio. This two-hour rule and the proximity of Palm Springs to Los Angeles resulted in a strong connection between the desert city and Hollywood in terms of history and culture (Palm Springs Bureau of Tourism, 2016). The Coachella Valley offers unique locations that are immediately recognizable and evoke audiences' emotions and memories, which appeals to producers. With one of the densest concentrations of mid-century modern architecture, identifiable landmarks, and some of the most beautiful and rugged landscapes in California, the Coachella Valley's scenery is always in demand. Combining these unique features with the public's renewed interest in the valley as a tourist destination results in the perfect motivation for taking Palm Springs film and television location and resource scouting into the twenty-first century.

\subsection{Web GIS}

Since the online location library is a web-based solution, no discussion of this topic is complete without some discourse about the definition of web GIS. In its infancy, web GIS was defined as being GIS that functions in a web browser. Research indicates, however, that the term requires broader definition. This section explores what web GIS is which naturally requires a brief discussion of the Internet and the World Wide Web (WWW) since web GIS would not be possible without them. Following the coining of the term geographic information systems by Roger Tomlinson in 1962, GIS is widely used and has become an indispensable tool.

In 1969, the U.S. Department of Defense Advanced Research Project Agency (ARPA) connected four physically separate computers, enabling communication between 
them. These nodes, located on university campuses, established an interconnected network of physically separate computers - the precursor to the Internet. The goal of this network, called the ARPANet, was to ensure that all nodes could continue communicating with one another even if one or more nodes were destroyed in a nuclear attack (Fu \& Sun, 2011). It would be more than 20 years before the invention of the World Wide Web, which revolutionized computer communications and set the foundation for the evolution of web GIS.

By 1990, Tim Berners-Lee, a researcher at the European Organization for Nuclear Research (CERN), was looking for a way to share documentation with his peers and other researchers doing similar work around the world. Using the worldwide network of connected computers, Berners-Lee invented the technologies that form the backbone of the web Hypertext Transfer Protocol (HTTP), Hypertext Markup Language (HTML), and the Uniform Resource Locator (URL). These new networked-based technologies enabled the global interconnection of documents and pages now known as the World Wide Web, paving the way for web GIS.

Nearly every industry that needs to track the location of something, or somebody, or to analyze the spatial relationship between things, has benefited from the popularity of GIS technology. As the web has grown and matured in its robustness and capabilities, GIS taken advantage of this growth. In 1993, Xerox Corporation Palo Alto Research Center (PARC) demonstrated the first online map viewer running over the Internet's World Wide Web, ushering in the very beginning of web GIS.

There are numerous reasons why GIS has evolved into being a technology delivered more and more over the web. For instance, GIS has a global reach and can facilitate a much larger number of users than desktop GIS can. The World Wide Web opens up GIS tools and technologies to any user with an Internet-connected computer and browser or mobile device. Since web GIS may be accessed using a standard Internet web browser, it offers greater cross-platform capability and can run on PC, Mac, and other computer operating systems. The ubiquity of handheld devices and mobile phones is also fueling the transformation of desktop GIS into a web-based technology. Nearly every mobile phone maker includes some type of Internet browser on even the most basic smartphones. In fact, it is this Internet connection and web browsing capability that define the class of mobile phones called smartphones. Developers of web GIS platforms are very aware that large numbers of novice users are flocking to web GIS platforms. This drives development of software that is more user-friendly than traditional desktop software, enabling users to perform advanced visualization and spatial analysis without having extensive education or training.

If there is one disadvantage to web GIS, it is its distributed nature. A typical user of a web GIS platform uses a computer or mobile device to connect to one or more geographically separated computers over the Internet or even cellular data networks. This distributed communication is affected by the speed of the device or network connection and the performance of the computers on both ends. A fast and reliable internet connection is critical to the success of web GIS. Slow communication may result in poor web GIS performance.

This section began with the narrow definition of web GIS as being GIS accessed in a web browser. As the chapter continued, handheld devices and mobile smartphones were introduced as platforms for web GIS. The evolution of web GIS includes the creation of 
native apps running on handheld devices that no longer require a web browser. Today, desktop GIS software also supports the use of web-based GIS data sources. With advancement in both web and GIS technology, the original definition is too narrow and does not account for when GIS tasks are performed on a desktop or in a mobile application. It might be better to define web GIS as any GIS that uses web technologies to communication between components (Fu \& Sun, 2011).

\subsection{Summary}

Chapter 2 explored some of the history of Hollywood and the studio system in the context of local studio-only film production. This chapter established definitions for terms including runaway production and location scouting, and it provided some historical context for the evolution of runaway productions in California and the rise of on-location production. Economic impacts of runaway productions were explained as were ways that governments have worked, through tax incentives and film commissions, to turn the tide of economic losses from productions leaving the state. The chapter also provided some background on the development of the Internet and World Wide Web, upon which web GIS evolved and enabled the development of the online location library.

Having established that existing location scouting tools are inferior and do not meet the needs of regional film offices, the remaining chapters will address the design, implementation, and outcomes of deploying a web-enabled, map-based online location library. 


\section{Chapter 3 - Systems Analysis and Design}

An essential part of successful project planning includes collecting the client's business requirements. Section 3.1 lists the formal functional and nonfunctional project requirements gathered through numerous meetings and discussions with the client. Combining the preliminary understanding of the problem with detailed requirements provides a natural path toward defining an introductory solution design. While progress toward any solution naturally results in changes to the original design, the basic system design and its components are outlined in Section 3.3. No good project can be expected to succeed without a plan. A project plan is a working document and changes with progress toward the final solution. Section 3.4 presents one snapshot of the project plan.

\subsection{Problem Statement}

The client's current process for identifying film and television production locations is time-consuming and often requires cumbersome coordination between other film offices and location scouts. The current location scouting process can be slow, and tight production turnaround times can result in lost projects and money. This project addressed these challenges as well as ones in finding local talent and film-friendly businesses. Putting these components together into a single search tool with an intuitive interface would allow the client to focus his limited time on more complex requests. Providing a self-service search and filter tool for routine inquiries would allow the client to work more efficiently and provide location details quickly.

\subsection{Requirements Analysis}

The key to any successful development project is to ensure a thorough understanding of the client's needs. Significant time was spent in the initial analysis phase of the project so that both the client and developer understood and agreed on the project scope. Their agreement clarified which features and functions were to be included, the client's expectations in terms of system performance, and what minimum systems specifications could still provide optimal operation and support. The project team, in consultation with the client, established a set of functional and nonfunctional requirements.

\subsubsection{Details of Functional Requirements}

Table 1 lists the general statement of the functional requirements, and Section 3.2.1 discusses each requirement in detail. The functional requirements listed in this section address the specific features that the solution will provide.

Table 1. Online Location Library Functional Requirements

\begin{tabular}{|l|l|}
\hline Functional Requirement & Description \\
\hline $\begin{array}{l}\text { Searching by Attribute and } \\
\text { Filtering by Type }\end{array}$ & $\begin{array}{l}\text { Users shall be able to search and filter location } \\
\text { attributes using multiple methods. }\end{array}$ \\
\hline
\end{tabular}




\begin{tabular}{|l|l|}
\hline Functional Requirement & Description \\
\hline Mp and Listing Details Pop- & $\begin{array}{l}\text { Clicking on a map icon or a location listing shall } \\
\text { display a pop-up with relevant details for the } \\
\text { location. }\end{array}$ \\
\hline Display Permit Requirements & $\begin{array}{l}\text { Location pop-ups shall display permit } \\
\text { requirements. }\end{array}$ \\
\hline Update Attributes & $\begin{array}{l}\text { After successful authentication, the application } \\
\text { administrator shall be able to update permit } \\
\text { requirements by jurisdiction. }\end{array}$ \\
\hline Media (photo/video) Display & $\begin{array}{l}\text { Thumbnail images shall appear under the location } \\
\text { type tabs in the left pane of the map display. } \\
\text { Images will also appear in location pop-ups. } \\
\text { Clicking on an image shall display a full-size } \\
\text { image. }\end{array}$ \\
\hline $\begin{array}{l}\text { Crew, Talent, and Vendor } \\
\text { Display }\end{array}$ & $\begin{array}{l}\text { Film-friendly businesses and other service } \\
\text { providers shall display on the same map as } \\
\text { production locations. }\end{array}$ \\
\hline Create New Record & $\begin{array}{l}\text { After successful authentication, an authorized user } \\
\text { shall be able to create new location, person, and } \\
\text { vendor records. }\end{array}$ \\
\hline
\end{tabular}

The Searching by Attribute and Filtering by Type requirement provides various methods for a user to search and filter locations that appear on the map. The Search By Attribute tool allows a user to type a word or phrase in a search box and to find locations in the library that match. The tool searches the location title, category, and description attributes of the Location feature class. Results matching the user's entry are displayed below the Search tool. When the user selects a matching record, the map zooms to the selected location, and a pop-up containing information about the location appears. If a search returns no matching results, the message "No matching results were found" is displayed. The Filter By Type tool allows the user to filter the map display so that only locations matching the selected category remain. The application displays location categories as separate tabs in an accordion container in the left pane of the interface. When the user selects a tab (labeled by category or interest), the map displays updates to reflect only locations in the database classified with the selected category. The All Locations pane, at the top of the interface, removes all filters and returns the map display to the default showing all locations.

When a user clicks a location icon on the map, a pop-up window displays the relevant details of the location. Each pop-up contains information about the location including production details and contact information. The pop-up also contains allows the user to zoom to the location. 
Permit requirements information is also included in the pop-up. The pop-up windows have navigation buttons that allow the user to move between location and permit details. The online feature layer, Jurisdiction, contains the permit details for each city, and an authorized administrator may update the permit requirements for any given jurisdiction. Since permit data is stored in its own separate hosted feature layer, there is no requirement for the administrator to update individual locations to reflect the latest permitting requirements.

The client classifies locations in the library by category. The solution displays each location category on a separate tab in the accordion container on the left side of the interface. After the application loads, titles of each location appear under the appropriate category heading. Clicking the location title under the category heading displays the location information and permit pop-ups. The thumbnail image displayed in the pop-up is also a hyperlink that opens a new browser tab to displays full-size images of the location.

One dataset provided by the client included film-friendly businesses and other service providers frequently hired by film and television productions. An important feature of the location library is the display of service providers that are near the currently selected location. While exploring a location, the user can see icons representing each of these businesses on the map. By displaying locations and businesses on the same map, a user determines the likelihood that the area can support the production.

Some jurisdictions offer to reduce or waive film and television production approval fees. The purpose of offering these incentives is to bring in more productions and the positive economic impacts that accompany them. The online location library prominently displays any available incentive information in the Permit Information pop-up.

The Submit New feature was requested by the client to allow the addition of new location, people, and vendor records to the location library. When an authorized user successfully logs into the system, an entry form is available for capturing and submitting the new data or updating existing records. All required fields for the new entries are clearly marked. The application allows the client to prevent new features from being displayed until the administrator has finalized the records. The review process helps maintain ongoing database integrity and ensures that the user correctly filled out all necessary data entry fields.

\subsubsection{Details of Nonfunctional Requirements}

Table 2 provides a general description of nonfunctional requirements. Unlike functional requirements, nonfunctional requirements do not address the features or operation of the application. Instead, they describe the technologies used to implement the solution and deliver the functions, as well as any system performance requirements and hardware or software prerequisites. Nonfunctional requirements may also include the specific, measurable performance goals of the project or application. In the case of the online location library, nonfunctional requirements specify performance goals the client requires once the application is operational. For the GPSFA, this included increasing the average number of locations booked in a month. 
Table 2. Online Location Library Nonfunctional Requirements

\begin{tabular}{|l|l|}
\hline Name & Description \\
\hline $\begin{array}{l}\text { Combine Datasets into } \\
\text { Solution Architecture }\end{array}$ & $\begin{array}{l}\text { The online location library shall bring together location, } \\
\text { person, and vendor datasets into a single, map-based } \\
\text { solution. }\end{array}$ \\
\hline Browser Compatibility & $\begin{array}{l}\text { The application shall be built using JavaScript, } \\
\text { Cascading Style Sheet, HTML5, and Esri ArcGIS API } \\
\text { for JavaScript 3.x. }\end{array}$ \\
\hline $\begin{array}{l}\text { The application shall be a web-based application } \\
\text { compatible with Microsoft Internet Explorer 11 and } \\
\text { higher and Google Chrome version 50.0 and above. }\end{array}$ \\
\hline Scouting Time & $\begin{array}{l}\text { The application shall speed up the location scouting } \\
\text { process and reduce the amount of time the client spends } \\
\text { on individual locating scouting requests. }\end{array}$ \\
\hline
\end{tabular}

One of the primary motivations for this project was the client's desire to make his location scouting process easier and less time consuming. This goal is partially achieved by specifying the nonfunctional requirement that the project bring multiple datasets together into one place for easier use. The datasets include the client's lists of film and television production locations, industry professionals for hire, and other businesses and service providers that support film and television production. Bringing all three of these datasets into a single location with one interface greatly streamlining the location scouting processes.

Early in the project analysis phase, the client agreed that a custom web-based application would meet his requirements. The application was built using web programming technologies including JavaScript, Cascading Style Sheet (CSS), HTML5, and Esri ArcGIS API 3.18 for JavaScript.

Since there are competing web browsers in various versions, the client agreed to a minimum version to ensure that the location library functions as intended and gives the client the best chance to meet his performance goals. The agreement between the client and the project team was that the application function on browsers compatible with Microsoft Internet Explorer versions 11 and higher and Google Chrome version 50.0 and above.

The final nonfunctional requirement documents the intended performance improvement goals. As discussed earlier, the client's inefficient scouting process sometimes would result in losing projects. By developing a self-service location scouting tool, the overall process takes less time and reduces the amount of effort the client spends on individual requests. By providing production details and requirements in response to the initial inquiry, the client can improve his efficiency and increase the overall number of bookings. The client will no longer waste time researching locations that could not accommodate a particular production due to size or other reasons. 


\subsection{System Design}

The Online Location Library is a stand-alone custom JavaScript web application. The application utilizes the Esri ArcGIS Online cloud GIS and ArcGIS API for JavaScript. The four major components that make up the application are ArcGIS for Desktop, ArcGIS Online, the client's web server, and the end user's computer running a supported web browser. Figure 3-1 provides a graphic overview of the components, how they connect, and the flow of data from the various components to the user's browser.

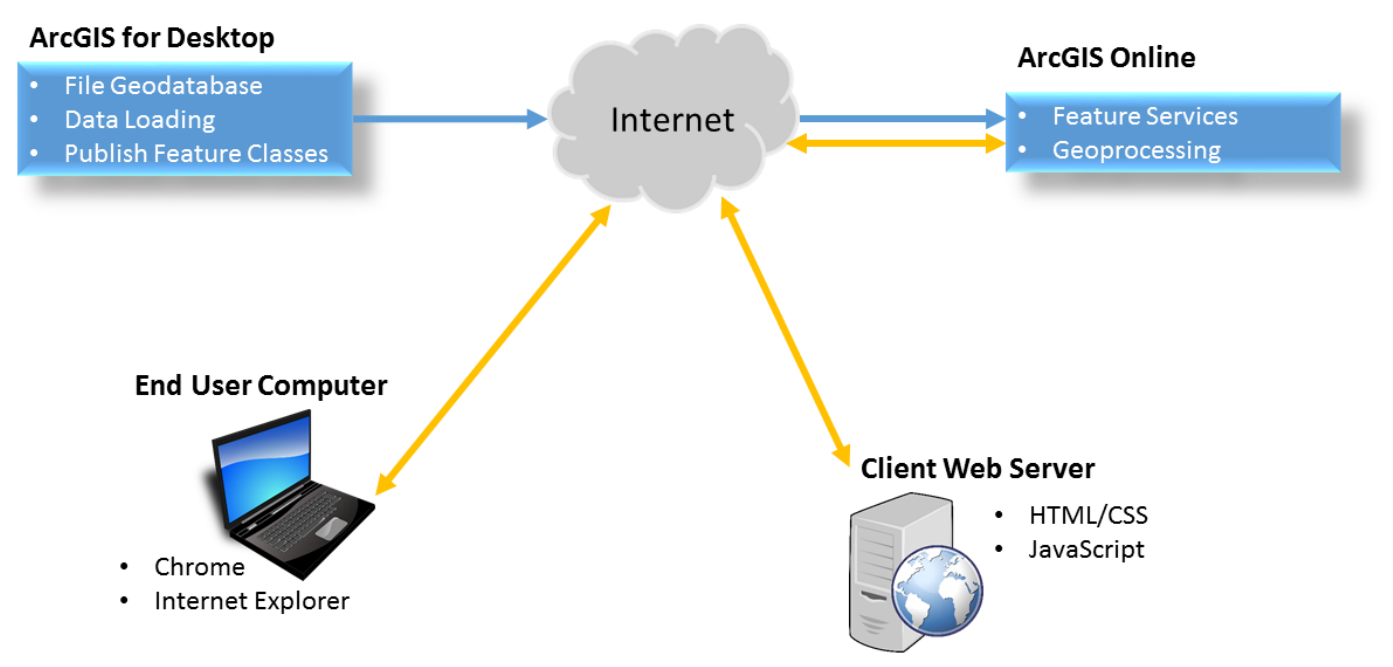

Figure 3-1: Graphic representation of application components

Hosted on the client's existing web server, the application uses HTML, CSS, and JavaScript. ArcGIS API 3.18 for JavaScript and ArcGIS Online provide GIS functionality in the solution. The project team developed the web application to be compatible with specific web browsing software. The browser requirement was Internet Explorer version 10 and Google Chrome version 50.

The ArcGIS for Desktop suite was used to create a file geodatabase for loading the client's data. The design for the feature classes separated each of the three datasets into an individual feature class. The design of each feature class's attribute schema was a collaboration between the client and the project team. Once all feature classes were developed, the entire collection of data were published as a feature service hosted by ArcGIS Online. This step allows the online feature services to be programmatically accessed as Representational State Transfer (REST) endpoints for use in the JavaScript web application. Once published as a hosted feature service, the feature classes in the original file geodatabase were no longer needed for implementing the web application. The process of publishing the data in ArcGIS Online copies the data to ArcGIS Online.

The JavaScript programming language and Esri ArcGIS API for JavaScript were used to create the web application. The map and the application presentation layer were built using standard web programming techniques and patterns. The web application 
takes advantage of ArcGIS API for JavaScript to provide all the necessary code and methods for accessing the online feature services as well as the mapping functionality.

\subsubsection{System interface requirements}

As a stand-alone application, interfaces with other systems are minimal. Both the client and partner organizations host their location-related pictures and media files on publically accessible web servers. For displaying images of locations, the location library directly references these systems, using web protocols to retrieve and display any media files. Esri hosts and maintains ArcGIS API for JavaScript in the cloud. The programming team used web-based JavaScript coding patterns to access the hosted GIS functionality.

\subsubsection{Communication requirements}

According to the client, the GPSFA website currently receives approximately 1,600 visitors each month during the peak location-scouting season. Client goals include increased website traffic, with 3,000 visitors each month in 2017, then an increase of 18 $\%$ each year thereafter. The online location library is a key component of meeting these goals and servicing the increased traffic. It may be necessary for the client to upgrade services to accommodate the increased web traffic and data throughput.

Being a web-based solution, the online location library communicates via standard HTTP protocol and Internet protocol (IP) communication. The client currently contracts with a third party for web server hosting and maintenance services. In the event of issues with server performance, the client will address these issues with the service provider.

\subsubsection{Hardware and software requirements}

The project used standard hardware and software for developing the web solution as well as for hosting and maintaining the completed web application. The development team used ArcGIS 10.4 for Desktop to organize and manage the operational layers that would be used in the web application. A standard Dell M3800 laptop running the Windows 7 64-bit operating system was the development platform. The WebStorm Integrated Development Environment (IDE) was the JavaScript platform used by the development team, and current versions of Google Chrome and Internet Explorer web browsers were used for solution testing. The client's hosted web server is a CentOS 6 64-bit Windows Server with a user-accessible control panel.

\subsubsection{Policies and standards}

The client did not specify existing policies or standards for project implementation and interface design. The programming team followed industry best practices for HTML5 and JavaScript web development. The developer incorporated colors in the application design to compliment the client's current color scheme. The GPSFA logo was used where possible for a consistent look and feel of the client's brand. By following web design and JavaScript programming best practices, the developer ensured the greatest likelihood for compatibility, stability, and longevity of the solution. 


\subsection{Project Plan}

During the planning phase, the project team met with the client in order to understand the business of location scouting and the current workflow. The outcome of these discussions was a list of application requirements that the project team used to develop an initial project plan outlining all the phases, milestones, and tasks necessary to deliver the solution. Table 3 is the preliminary project plan, including estimated durations and proposed schedule.

\section{Table 3. Preliminary Project Plan}

\begin{tabular}{|c|c|c|c|c|}
\hline WBS & Task Title & $\begin{array}{c}\text { Start } \\
\text { Month }\end{array}$ & $\begin{array}{c}\text { End } \\
\text { Month }\end{array}$ & $\begin{array}{c}\text { Budgeted } \\
\text { Hours }\end{array}$ \\
\hline 1 & Planning & & & \\
\hline 1.1 & Determine/Document requirements & April & May & 60 \\
\hline 1.2 & Write MIP proposal for faculty and client approval & April & April & 20 \\
\hline 1.3 & Develop project plan & April & April & 16 \\
\hline 1.4 & Identify ArcGIS Online hosting site & April & April & 4 \\
\hline 1.5 & Determine WordPress integration need/feasibility & April & April & 4 \\
\hline 1.6 & Client sign-off of requirements & July & July & 0 \\
\hline 2 & Design & & & \\
\hline 2.10 & Design \& document conceptual data model & May & June & 8 \\
\hline 2.15 & Design \& document physical data model & July & July & 2 \\
\hline 2.20 & Document use cases & May & May & 8 \\
\hline 2.40 & Receive photos/videos/attributes from client & May & June & 0 \\
\hline 2.50 & Create photos/videos if necessary & May & June & 0 \\
\hline 2.60 & Obtain alliance member database & May & June & 0 \\
\hline 2.70 & JavaScript self-training & June & Aug & 0 \\
\hline 2.80 & Esri JavaScript API training & July & July & 25 \\
\hline 2.10 & Wireframe web app & July & July & 20 \\
\hline 3 & Implementation/Testing & & & \\
\hline 3.10 & Prepare member database if necessary & July & Aug & 0 \\
\hline 3.20 & Upload photo and video media if necessary & July & Aug & 0 \\
\hline 3.30 & Review \& prepare client data & July & Sept & 40 \\
\hline 3.35 & Build geodatabase & Aug & Aug & 8 \\
\hline 3.40 & Publish feature services in ArcGIS Online & July & Sept & 8 \\
\hline 3.45 & Create web map & Aug & Aug & 8 \\
\hline 3.50 & Build JavaScript application & July & Sept & 160 \\
\hline 3.60 & Test JavaScript application & Sept & Sept & 40 \\
\hline 3.70 & Rework JavaScript application & Sept & Sept & 20 \\
\hline 3.80 & Integrate mapping application into client's website & Sept & Sept & 10 \\
\hline 4 & Deployment & & & \\
\hline 4.05 & Determine final hosting (CSS or CVEP) for feature services & Oct & Oct & 2 \\
\hline 4.10 & Migrate feature services to final hosting & Oct & Oct & 0 \\
\hline 4.15 & Migrate JavaScript to client webserver & Oct & Oct & 8 \\
\hline
\end{tabular}




\begin{tabular}{|c|l|c|c|c|}
\hline 4.20 & Test app outside development group & Oct & Oct & 8 \\
\hline 4.30 & Revise app based on client feedback & Oct & Oct & 40 \\
\hline 4.40 & Share app with ArcGIS Online group everyone & Oct & Oct & 0 \\
\hline 4.50 & Final client review \& sign-off & Oct & Oct & 4 \\
\hline 5 & Project Close & & & \\
\hline 5.10 & Finalize solution documentation & Oct & Oct & 16 \\
\hline 5.20 & Deliver updated artifacts to client and CVEP & Oct & Oct & 0 \\
\hline 6 & MIP Report \& Defense & & & \\
\hline 6.10 & Draft MIP report & Oct & Oct & 80 \\
\hline 6.1 .1 & Chapters 1 \& 2 to editor & Aug & Aug & 20 \\
\hline 6.1 .2 & Revisions to chapters 1 \& 2 & Sept & Sept & 8 \\
\hline 6.1 .3 & Chapters 3 \& 4 to editor & Sept & Sept & 20 \\
\hline 6.1 .4 & Revisions to chapters 3 \& 4 & Oct & Oct & 8 \\
\hline 6.1 .5 & Chapters 5 \& 6 to editor & Oct & Oct & 20 \\
\hline 6.1 .6 & Revisions to chapters 5 \& 6 & Oct & Oct & 8 \\
\hline 6.1 .5 & First draft to advisor & Oct & Oct & 0 \\
\hline 6.20 & Draft MIP Defense Presentation & Nov & Dec & 60 \\
\hline 6.2 .1 & Advisor reviews defense presentation & Nov & Nov & 40 \\
\hline 6.2 .2 & Finalize defense presentation & Nov & Nov & 40 \\
\hline 6.30 & MIP defense & Nov & Dec & 8 \\
\hline 6.40 & Prepare \& submit MIP CD & Dec & Dec & 8 \\
\hline 6.50 & Submit MIP report to Armacost Library & Dec & Dec & 2 \\
\hline & & & Total & 861 \\
\hline
\end{tabular}

\subsubsection{Planning Phase}

During the planning phase, interviews were conducted with the client to help the project team understand the current location scouting workflow. The goal of these interviews was to document the current location scouting workflow and begin collecting information on the client's pain points in the existing processes. This phase was also an opportunity for the project team to understand the film and television location scouting business. Learning a new vocabulary was necessary to improve communication with the client and industry partners as well as to prepare for the research ahead. The outcomes of this phase were detailed functional requirements, design drafts, and a project plan. This was the longest phase of the project, and some aspects of project scoping and business process review continued well into the early stages of project development.

\subsubsection{Design Phase}

The design phase included preliminary design work on the file geodatabase and feature class structure. To ensure the necessary attributes were captured in the datasets, the client was regularly consulted. One significant challenge during this phase was getting pictures for locations. Although not formally scheduled for delivery until phase three, concerns about the location pictures and datasets resulted in the project team requesting these data earlier than planned. By executing this step earlier in the timeline, padding was created in 
the schedule in the event that the client had to go out into the field to collect the necessary data and pictures. This proactive planning helped to prevent falling behind schedule.

\subsubsection{Design Phase}

It was during this phase that the project team requested the three datasets from the client. The client had originally anticipated working with industry partners to collect data and pictures from locations throughout the study area. Unfortunately, the partners were unable to share their location data and pictures. At this point, the project implemented the first contingency plan. Since the partner agencies did not provide their data and images, the client selected 10 representative locations throughout the Coachella Valley, created the necessary data attributes, and provided pictures of these sample locations. Time was lost waiting for the images and data that affected the timeline for this phase. One other significant milestone for this phase was the Esri instructor-led training in the use of ArcGIS API for JavaScript. While experienced in programming, the development team had minimal experience in web design and programming and no experience in web development using ArcGIS API for JavaScript. The Esri public training schedule drove the timing of this critical training task and was beyond the control of the project team.

\subsubsection{Implementation/Testing Phase}

The majority of the JavaScript development work occurred in the implementation/testing phase. As the web application development progressed, the project team uncovered issues with the original feature class design. The altered geodatabase design was incorporated into the solution, and the feature service was republished in ArcGIS Online. Python scripting automated some of the manual tasks of creating and assigning feature class domains and subtypes. Some level of design iteration on the geodatabase had been anticipated, and using the Python scripts to rebuild the geodatabase during testing helped minimize time lost during this phase.

\subsubsection{Deployment Phase}

During the deployment phase, the application code files were migrated to the client's website. The project team had a choice to host the feature services on the University of Redlands ArcGIS Online organizational account or migrate them to the Coachella Valley Economic Partnership (CVEP) site. In an attempt to make up lost time, it was decided to keep the feature services on the university ArcGIS Online site.

The final phases of the project included completing all required documentation. Documentation included artifacts required for the MS GIS program as well as documentation and instructions for the client for adding and managing location library records.

\subsection{Summary}

Chapter 3 laid out the project requirements analysis, system design, and project plan portions of the project. The project team invested significant effort in documenting and clarifying the client's needs and developed solid business requirements for the online 
location library. The solution envisioned by the team to meet all requirements was an ArcGIS Online-based custom web application. This solution incorporated several Esri technologies including file geodatabase development, ArcGIS Online feature services, and ArcGIS API for JavaScript development. A sound project plan was developed and monitored to ensure progress. Due to an aggressive project scope, delays in receiving data, and other setbacks, there were significant delays in project implementation that ultimately affected the project deliverable. 


\section{Chapter 4 - Database Design}

Designing the database supporting the online location library was a key step in the overall project implementation. Among the most visible features of the location library were the location, permit, and vendor pop-ups accessible from the web map. These pop-up windows contain the critical decision-making information necessary for a director or location scout to make a determination about a site. Care was taken in reviewing which attributes to collect for location attributes to ensure that all necessary information was easily accessible.

This chapter covers the database design process, beginning with Section 4.1 and a discussion of the conceptual data model, which captures an idealized data design that documents all data elements and their relationships. Next, Section 4.2 describes the process of transforming the conceptual data model into a logical one that supports implementation in a geodatabase environment. Discussion of where the project data came from appears in Section 4.3, collection methods used are discussed in Section 4.4, and the steps used to prepare the data for a GIS are discussed in Section 4.5.

The client's original plan for data was to collaborate with other location managers and film offices to share existing databases. When data sharing agreements did not develop, the project relied on a contingency plan to develop a much smaller subset of location data to construct a proof-of-concept online location library.

\subsection{Conceptual Data Model}

The conceptual design for the database originally identified six entities: Location, Architectural Style, Asset, Person, Position, and Vendor. Only three of these entitiesLocation, Person, and Vendor-ended up in the logical design, for reasons explained in this section. One of the primary goals of the project was to efficiently get location information into the hands of film and television professionals. Therefore, location records were the cornerstone of the database design. Discussions about the original design of the location database resulted in the classification of locations with one or more architectural styles and assets that would be of interest to filmmakers. Oftentimes when location scouts are searching for a viable site, they already have a vision for a scene and they describe it in terms of its architectural style, the view, or the buildings. For example, a residential home might be described as having Mediterranean or mid-century architectural style. Even the most casual user with a rudimentary understanding of architectural style can easily understand these descriptions. However, the identification of location assets as entities requires further explanation. In the context of this project, a location's assets included architectural design elements, physical amenities such as swimming pools or guesthouses, and even specific views from the location. By opening up the asset entity to store values such as a guesthouse or a secondary building, the possibility of more than one architectural style for a location exists. The main building on a site might be categorized as modern, but the guesthouse might be contemporary. This results in a location potentially having more than one architectural style. Figure 4-1 shows the conceptual entities and their relationships to one another. 


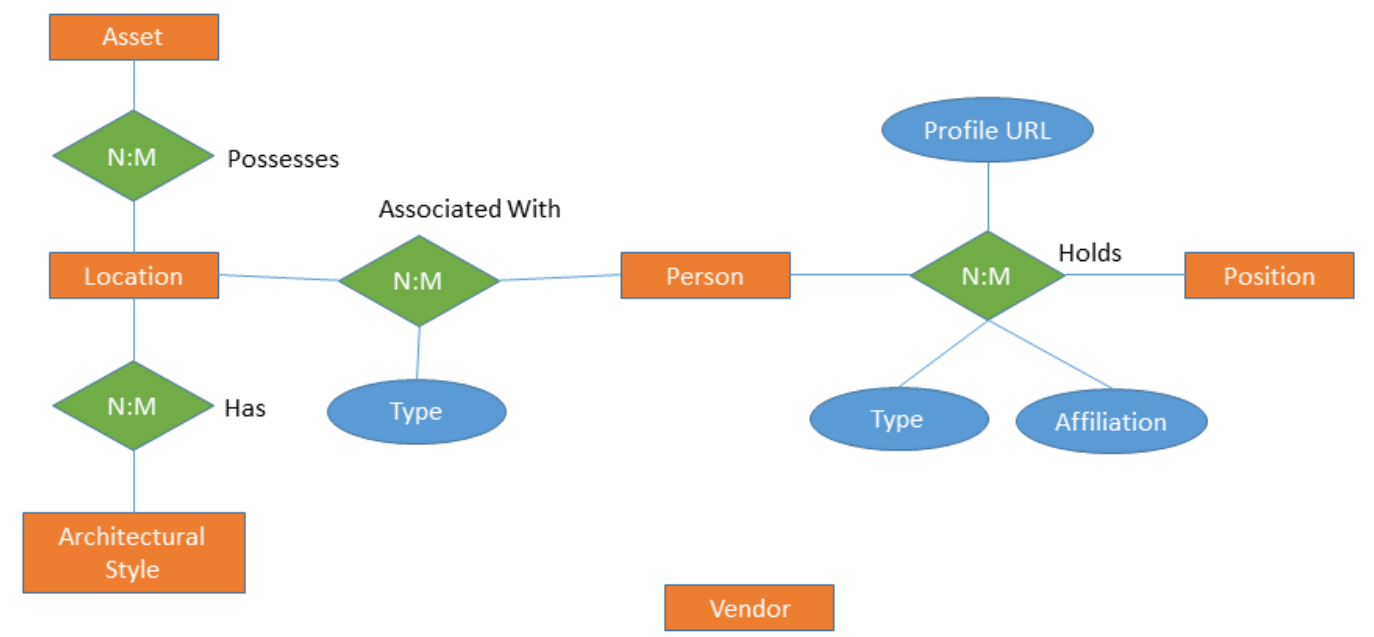

Figure 4-1: Location library conceptual data model

Similar to the relationship with architectural style and assets, the Location entity was also envisioned to have a relationship with one or more people, or a Person, entity. In the conceptual model, a Person entity associated with a location is classified by a particular role. This role, or person type, would include Owner, Point of Contact, or Location Manager. The Person entity served the secondary purpose of documenting records of film and television professionals available for hire. While not affiliated with a location, these Person entities would represent professionals and tradespeople commonly needed to support a film or television production. The Person entity, in this scenario, would be associated with a Position entity and various attributes describing the individual's professional profile.

The conceptual design captures the Vendor entity as a stand-alone entity. This data type would store records of businesses that support the production industry. Different from the Person entity, a vendor would be a service provider, such as equipment rental, catering, or security services, as opposed to the Person entity, which would include writers, directors, and actors.

\subsection{Logical Data Model}

Conversations with the client about mission-critical location attributes helped determine a database design for locations that was easily extended to include the Person and Vendor entities. Since no industry standard for cataloging location data was available for reference, the client suggested a straightforward design that would meet the organization's need for simplicity in management and operation. Some entities identified as part of the conceptual design were abandoned for a simplified data model that addressed the client's primary need for presenting location details to the user at the beginning of the scouting process.

The progression from conceptual database design to a physical implementation involved trial and error. With the client's requirement that the solution be a web-based application accessible via a supported browser, the decision was made to use ArcGIS Online as the final hosting environment for project data. While the data could have been 
loaded directly into ArcGIS Online, the design team initially used ArcGIS for Desktop to build the database in Esri file geodatabase format and then published the data in ArcGIS Online. This staged approach provided greater flexibility and convenience during the design phase, given the trial-and-error nature of the process. The general design of the file geodatabase, including feature classes, relationship classes, and nonspatial reference tables, appears in Figure 4-2.

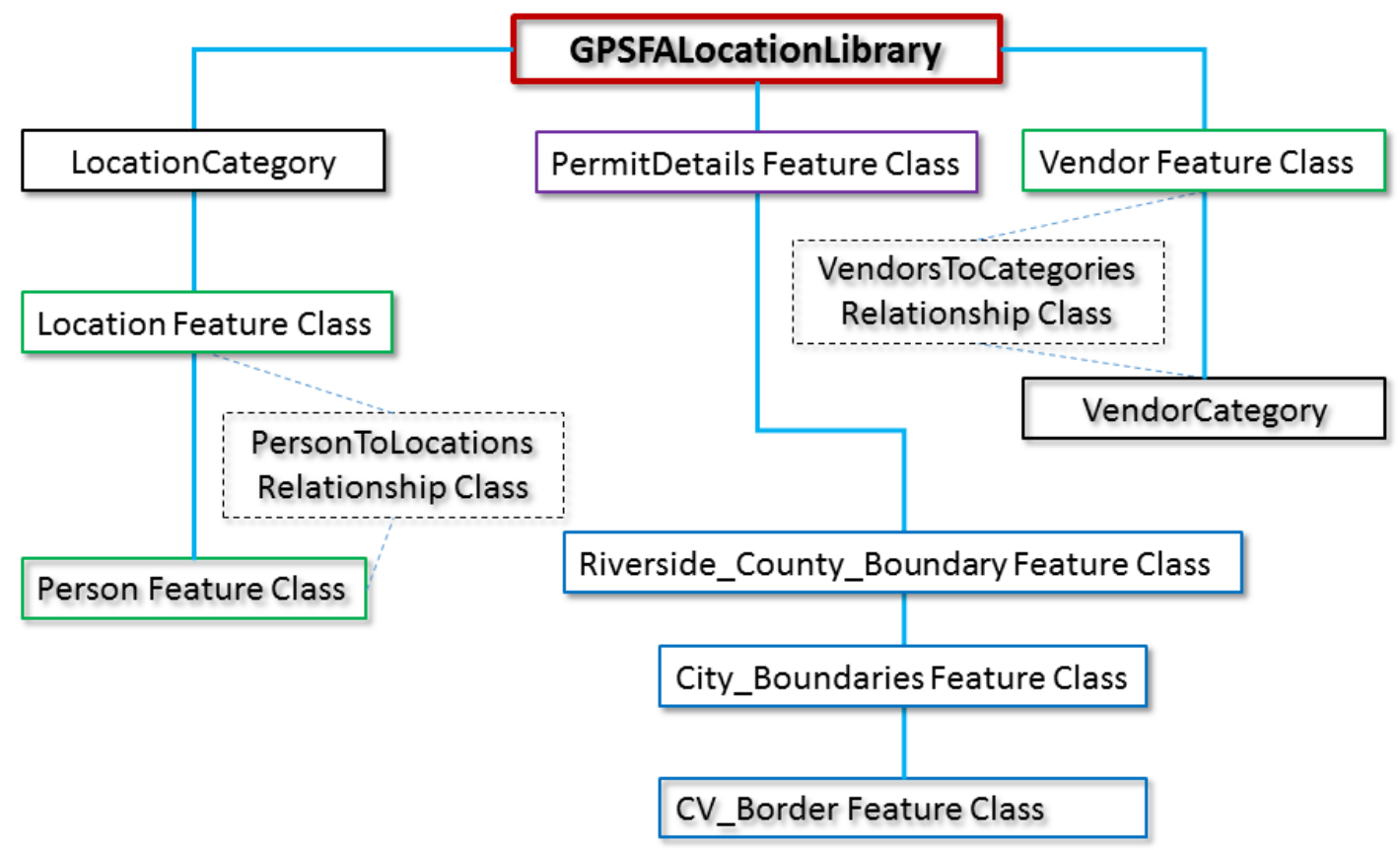

Figure 4-2: Geodatabase design indicating feature and relationship classes

\subsubsection{Location Feature Class}

The decision to create the logical database in ArcGIS for Desktop and publish in ArcGIS Online proved to be useful when working through various design scenarios. Some ArcGIS geodatabase features and functions, including subtypes and domains, are not easily removed once applied to a file geodatabase. Adopting an iterative design process using an XML export of the entire file geodatabase, including data, allowed the development team to make incremental design changes and perform testing since the team could easily re-create the entire geodatabase.

The location feature class was developed using an Esri geodatabase subtype with 41 different values on the location category attribute. Each subtype has a coded value domain associated with the type attribute, totaling 41 separate domains. Many of these domains contained 10 or more coded values. Creating and re-creating all the domains by hand would have been time-consuming and labor-intensive. Developing a Python script to automate their creation, and using the XML export to re-create everything from 
scratch, saved significant time and allowed for iterative database development and testing.

The Location feature class is the cornerstone dataset for this project. This point feature class contains all location-specific details, represents the core of the online location library, and stores the majority of the information that film professionals seek. Table 4 shows a sample of the key attributes in the Location feature class.

Table 4. $\quad$ Location Feature Class Key Attributes

\begin{tabular}{|l|l|}
\hline Key Attribute & Key Attribute Description \\
\hline LocTitle & $\begin{array}{l}\text { This is the title of the location and contains keywords for location } \\
\text { searches. It is one of the attributes used by the search widget. }\end{array}$ \\
\hline LocCat & $\begin{array}{l}\text { The location category classifies each location. There are 41 unique } \\
\text { categories, and this attribute was implemented as a geodatabase } \\
\text { subtype. It is one of the attributes used by the search widget. }\end{array}$ \\
\hline LocShortSum & $\begin{array}{l}\text { The location short summary is a short version of the location } \\
\text { description. It is one of the attributes used by the search widget. }\end{array}$ \\
\hline LocLongSum & $\begin{array}{l}\text { The location long summary is a longer, more detailed version of } \\
\text { the summary. }\end{array}$ \\
\hline
\end{tabular}

Provided with the location data from the client was a list of 41 location categories, including Accommodation, Airport, Community Center, and Movie Ranch. Each of these categories was further subdivided into anywhere from 1 to 36 location types. The location category attribute was implemented as a geodatabase subtype, and 41 subtype codes were set-up for each category. The benefit of this design was more advanced symbology when creating maps and using the subtype values for different location icons. While not implemented for this project, each of the location categories could have a different map icon so that the user could visually distinguish between location categories while viewing a map.

The client also provided individual lists of location types for each category. These lists were implemented as geodatabase domains on the location type attribute. The benefit of this part of the design was improved accuracy of data entry when adding locations. When a user adds a location and selects the category from the predefined list, a dropdown list appears, ensuring that the user selects a location type that correctly corresponds to the location category selected. An additional domain containing the values Yes and No was added to the active flag attribute for all three feature classes. The flag is used to determine when a new record is ready to be displayed in the library.

During the planning phase, the development team identified that locations in the Location feature class would have a relationship to people in the Person feature class. Specifically, individuals in the Person layer could take on the role of the Location Manager. Since this location-to-person relationship needed to persist beyond the ArcMap session, this relationship was implemented as a geodatabase relationship class, which enforces and persists relationships between different feature classes - in this case, between the Location and Person feature classes. The Relationship class was created as a simple type, as opposed to a composite type. This allows a person record to be removed 
from the feature class without also removing the location. The Relationship feature class identifies the location manager for each site. A person record in the feature class can manage no locations or several, but a location record in the feature class may only have no more than one manager. A simple Relationship class allows a particular person record to be removed from the Person feature class but does not also force the removal of the related location from the Location feature class. This design supports changes in location managers.

\subsubsection{Person Feature Class}

The Person feature class is another point feature class that stores records of people who manage or are the point of contact for booking a location. A person record may be associated with one or more locations. The details of the person, including name, address, contact information, and web address, are stored in this feature class. As previously discussed, this feature class participates in a simple relationship class with the Location feature class. Table 5 lists a few key attributes of the Person feature class.

\section{Table 5. Person Feature Class Key Attributes}

\begin{tabular}{|l|l|}
\hline Key Attribute & Key Attribute Description \\
\hline FName & First name of the person record; a required field \\
\hline LName & Last name of the person record; a required field \\
\hline Phone & $\begin{array}{l}\text { Contact telephone number for the person record; a required field } \\
\text { and a critical attribute for booking a location }\end{array}$ \\
\hline Email & $\begin{array}{l}\text { Contact email address for the person record; a required field and a } \\
\text { critical attribute for booking a location }\end{array}$ \\
\hline
\end{tabular}

\subsubsection{Vendor Feature Class}

The Vendor feature class stores records of businesses and service providers that deliver professional services likely needed in film and television production. Categories of vendor features include lodging, catering services, transportation, equipment rental, and aerial photography.

The client delivered a vendor dataset that indicated that more than one category could be applied to a vendor. This discovery resulted in the design decision to create a relationship class between the Vendor feature class and a nonspatial vendor category list. The category list was implemented as a nonspatial table without geometry. A simple relationship class was implemented with a many-to-many cardinality to support multiple categories assigned to one vendor. Table 6 lists a few key attributes of the Vendor feature class. 
Table 6. Vendor Feature Class Key Attributes

\begin{tabular}{|l|l|}
\hline Key Attribute & Key Attribute Description \\
\hline Name & $\begin{array}{l}\text { This includes the full name of the vendor or service provider. } \\
\text { Since vendor names are generally company names and not } \\
\text { individuals, the field was not broken down by first and last name. }\end{array}$ \\
\hline VenCat & $\begin{array}{l}\text { The Vendor category includes the service provided by the vendor. } \\
\text { This corresponds to the category in the nonspatial vendor category } \\
\text { table. }\end{array}$ \\
\hline VenPhone & $\begin{array}{l}\text { The phone number field is critical piece of contact information for } \\
\text { hiring the service provider. }\end{array}$ \\
\hline
\end{tabular}

\subsubsection{Permit Data Feature Class}

The Permit Data feature class is a polygon feature class that stores the geometry for the local agencies that issue filming permits in the Coachella Valley. These jurisdictions may be cities, counties, or tribal areas. Required filming permit details are stored in this feature class. Permit details include fees, processing time, incentives, and contact information for applying for a permit. Table 7 summarizes key attributes in the permit data feature class.

Table 7. Permit Data Feature Class Key Attributes

\begin{tabular}{|l|l|}
\hline Key Attribute & Key Attribute Description \\
\hline ProcessingTime & The anticipated time from permit application to notification \\
\hline PermitFees & The cost for a filming permit \\
\hline Incentives & Any incentives offered by the jurisdiction to encourage filming \\
\hline
\end{tabular}

When a user selects a location on the map, the web application presents both the location attributes and the permit attributes. This ensures that relevant information about the location and permits is presented to the user at the earliest part of the location scouting process. Historically, this critical decision-making information was often not discovered by the location scout until a filming permit application was submitted, which could negatively impact a production as a result - for example, the processing time not being accurately factored into the overall production schedule. Also, any incentives for filming should be presented to the user as early as possible in order to encourage the end user to book the location.

The Permit Data feature class was created by spatially combining several different polygon layers representing various boundaries. A polygon layer containing Riverside County data was obtained for storing permit information for sites administered by the county. A polygon layer of the cities of the Coachella Valley represented in the pilot dataset was used for storing the specific film permit attributes for each of the involved cities. The boundary of the Coachella Valley was used in defining the study area for the application. A subset of the Riverside County cities, called the study area, was created 
using the Coachella Valley boundary and Riverside County city boundaries. The Riverside County boundary was included as part of the study area since some client locations within the study area are managed by Riverside County and governed under its film permit requirements.

\subsubsection{Supporting Nonspatial Tables and Relationship Classes}

In addition to the four feature classes, the file geodatabase contained four nonspatial tables and relationship classes. The tables named LocationCategory and VendorCategory are support tables used by the web application for data lookup; the tables named PersonToLocations and VendorToCategories are the relationship classes previously discussed.

The LocationCategory table contains the category code and description attributes used in the LocCat attribute of the Location feature class. The web application uses this table to look up the location category description when building the accordion-style panes of the location filter feature.

The VendorCategory table stores the category code and description used in the VenCat attribute of the Vendor feature class. The creation of the table allows for future implementation of a vendor filter feature similar to the location filter feature.

PersonToLocations and VendorToCategories are relationship classes created in ArcMap. PersonToLocations relates specific in the Location feature class data to Person feature class records pertaining to the manager or point of contact for the location begin considered. VendorToCategories relates each vendor's data to one or more of the vendor categories.

\subsection{Data Sources}

The client provided operational data for locations, persons, vendors, and permits. The Coachella Valley boundary polygon layer came from the Coachella Valley Economic Partnership. City and county boundary polygons were provided by Riverside County.

All operational data came from the client in Microsoft Excel format and included full addresses. These data had no geographic or projected coordinate system at delivery. Data for Riverside County cities and the county boundary came from the Riverside County GIS portal. These data were received in North American Datum 1983 State Plane California (NAD83 SP CA) Zone 6, as was the Coachella Valley boundary polygon layer obtained from CVEP.

\subsection{Data Collection Methods}

Data collection was minimal on the part of the project team. The client collected all operational data for locations, persons, and vendors but did not deliver a dataset of filming permit requirements for each of the jurisdictions in the study area. The project team manually collected permit data from the GPSFA website. The process for collecting these data was copy and paste them from the website directly into the PermitDetails feature class in ArcMap. 


\subsection{Data Scrubbing and Loading}

Real-world data needed for any new GIS project are rarely provided in a usable format, and this project was no different. The sampling of location data delivered by the client was a relatively small dataset but still required effort to reformat and prepare it for loading due to special formats included in the Excel spreadsheet. The person dataset required minimal cleaning. The vendor data comprised a larger dataset and had unique formatting hurtles to overcome.

The location, person, vendor, and location category data were received from the client in a single spreadsheet on individual tabs. Since data would be loaded using a Python script, each dataset was split into a stand-alone spreadsheet. All datasets required corrections to column titles to adhere to ArcGIS for Desktop standards for attribute titles. Data values in all datasets were processed to remove leading and trailing spaces from the values. Additionally, a spell check was performed on all datasets to correct typos.

Also, the original data spreadsheet was linked to a client spreadsheet that was not included in the original delivery, but resulted in repeated error messages when opening the Excel file. This linkage had to be removed from the spreadsheet in order to allow moving forward with further review and scrubbing.

The client hired an outside contractor to collect and input data for locations into the Excel spreadsheet. To make this process more efficient and minimize typos, a custom drop-down field was created by the client and the contractor for location type, category, and architecture, and it was linked to a list of values on another portion of the spreadsheet. These spreadsheet customizations had to be removed while preserving the original values in each of the three attribute fields. This extra step added to the complexity of the data cleanup and reformatting steps.

Options for creating the 41 geodatabase domains included manually creating them and typing the values by hand, or developing automation to do it programmatically. A Python script was developed to create and populate these 41 domains. Each location category and its list of types had to be moved to its own spreadsheet tab and saved as an individual comma-separated value (CSV) file for the automation. This required customization using Microsoft Excel Visual Basic for Applications (VBA) in order to avoid having to save each of the newly created tabs manually as separate delimited text files. The text files were later loaded into the geodatabase as coded-value domains via a custom Python script.

All operational data from the client were delivered in a nonspatial format. For these data to be used in ArcMap, they first had to be geocoded using the Esri World Geocoding Service from ArcGIS Online. Since ArcGIS Online was the final destination for the project data, location records from Excel were added as an event layer in ArcMap then geocoded using the World Geodetic Survey (WGS) 1984 Web Mercator (auxiliary sphere) projection. This is the standard projection of content published in the ArcGIS Online environment. The process of geocoding from the event layer was repeated for the person and vendor datasets. Once the geocoding was completed and the data were loaded into their respective feature classes, a 100 percent visual quality-control check was performed on the Location layer. An ArcGIS Online image basemap and Google Maps were used to visually confirm that the geocoded point was correctly located. 
The vendor dataset was large and took extra time to review, clean, and load. The vendor records may have more than one vendor category associated to them. This was captured in the original Excel data as duplicated records. If the vendor record was categorized under three different categories, this meant that the record was entered into the spreadsheet three times, each time with a different category. The list of vendors had to be processed to remove the duplicates, and care had to be taken to ensure that the additional categories, if they existed, were not overlooked. Once the Vendor feature class was loaded, the VendorToCategory relationship class was used to associate the categories. Vendors associated with more than one category resulted in multiple records in the relationship class. Table 8 lists one example of a vendor records with three categories.

\section{Table 8. $\quad$ Example of Vendor with Multiple Categories}

\begin{tabular}{|l|l|l|}
\hline Vendor Category & Vendor Name & City \\
\hline Equipment Rentals & Brudvik Inc. Rental Division & Palm Springs \\
\hline Production Support Services & Brudvik Inc. Rental Division & Palm Springs \\
\hline Rentals_-Grip Equipment & Brudvik Inc. Rental Division & Palm Springs \\
\hline
\end{tabular}

Datasets that were not geocoded as part of this project included Riverside County city and county boundaries as well as a Coachella Valley boundary layer. These three shapefile datasets were projected into WGS 1984 Web Mercator (auxiliary sphere) to match the operational data.

\subsection{Summary}

While the final environment for the data associated with this project was ArcGIS Online, the decision was made to create and manage the data in a file geodatabase in ArcMap. Once the geodatabase was complete, the feature classes needed for the web application were published in ArcGIS Online and the geodatabase was retained as a reference copy. If design changes were needed, the file geodatabase and Python scripts allowed for a rapid redeployment of a clean geodatabase to test new design elements.

Of the four datasets used in the project, locations and vendors required the most review and scrubbing before loading. All datasets required some reformatting, including checking spelling. Data that were not geocoded for the project were projected into WGS 1984 Web Mercator (auxiliary sphere), the projection of ArcGIS Online content.

Creating 41 coded value domains for each of the location categories would have been very time-consuming if done by hand. A custom Python script was developed to automate the domain creation process. This automation streamlined the database development process and allowed for an iterative and incremental development workflow for the file geodatabase. 



\section{Chapter 5 - Implementation}

Once the database design was complete, implementation shifted to the programming of the custom JavaScript application. The application was developed in a three-tier approach that separates business logic from the presentation and data layers. The web solution is composed of three separate files: an HTML file, a JavaScript file, and a Cascading Style Sheet (CSS) file. This separation makes the code more readable and manageable. This chapter covers all three files and breaks down the components of the application in detail.

\subsection{Publishing the Hosted Feature Layer}

At the core of this web application are the feature classes and supporting tables published to ArcGIS Online. This group of data includes the location, person, vendor, and permit feature classes; the PersonToLocations and VendorsToCategories relationship classes; and the VendorCategory and LocationCategory cross-reference tables. These datasets needed to be published as Representational State Transfer (REST) services before they could be incorporated into the web application using JavaScript and ArcGIS API for JavaScript.

The datasets needed for the web application were created and configured using ArcMap as discussed in Chapter 4. Once created, the data were all published as a single feature service called OnlineLocationLibrary. Default parameters for the feature service were selected. Since the application requires new features to be added, the operations allowed on the service at the time of publishing were the following: create, query, and update. The delete operation was not enabled for this service in order to prevent any delete feature mistakes. Since an authorized user will have the ability to add and update features, mistakes may be corrected for both the location of features and any erroneous attribute values; therefore, delete capability is not necessary.

The relationship classes and cross-reference tables were published along with the feature classes. The relationship classes maintain the relations between location and person data between vendor and category. The cross-reference tables are used by the JavaScript application when creating the user interface to display location type descriptions on the accordion panes for the location filter feature.

\subsection{Developing the Web Application}

The user interface for the web application consists of a main pane, a header pane, and a left pane. The main pane contains the web map, which is key to the functionality of location library. This is the central visual element of the application and includes several standard map interaction tools. The header pane contains the client logo for branding purposes. The left pane contains a custom interactive location filter feature. The ArcGIS API for JavaScript formed the backbone of the GIS functionality of this project (Esri Inc., n.d.-a). Additional coding assistance was gathered from the samples published online from Esri (Esri Inc., n.d.-b). 


\subsubsection{Presentation Layer with Dojo}

The presentation layer of the application was created using Dojo Toolkit. Dojo Toolkit is a JavaScript framework that saves time with JavaScript web development by providing tools, such as language utilities and user interface components that are designed to work together. The toolkit is included as part of ArcGIS API for JavaScript ("Dojo Toolkit," n.d.). The application layout was designed and implemented prior to adding any mapping functionality. The theory behind this approach was to complete the difficult part of laying out the design of the interface without having to simultaneously focus on the mapping functionality. Figure 5-1 shows the application interface and its components.

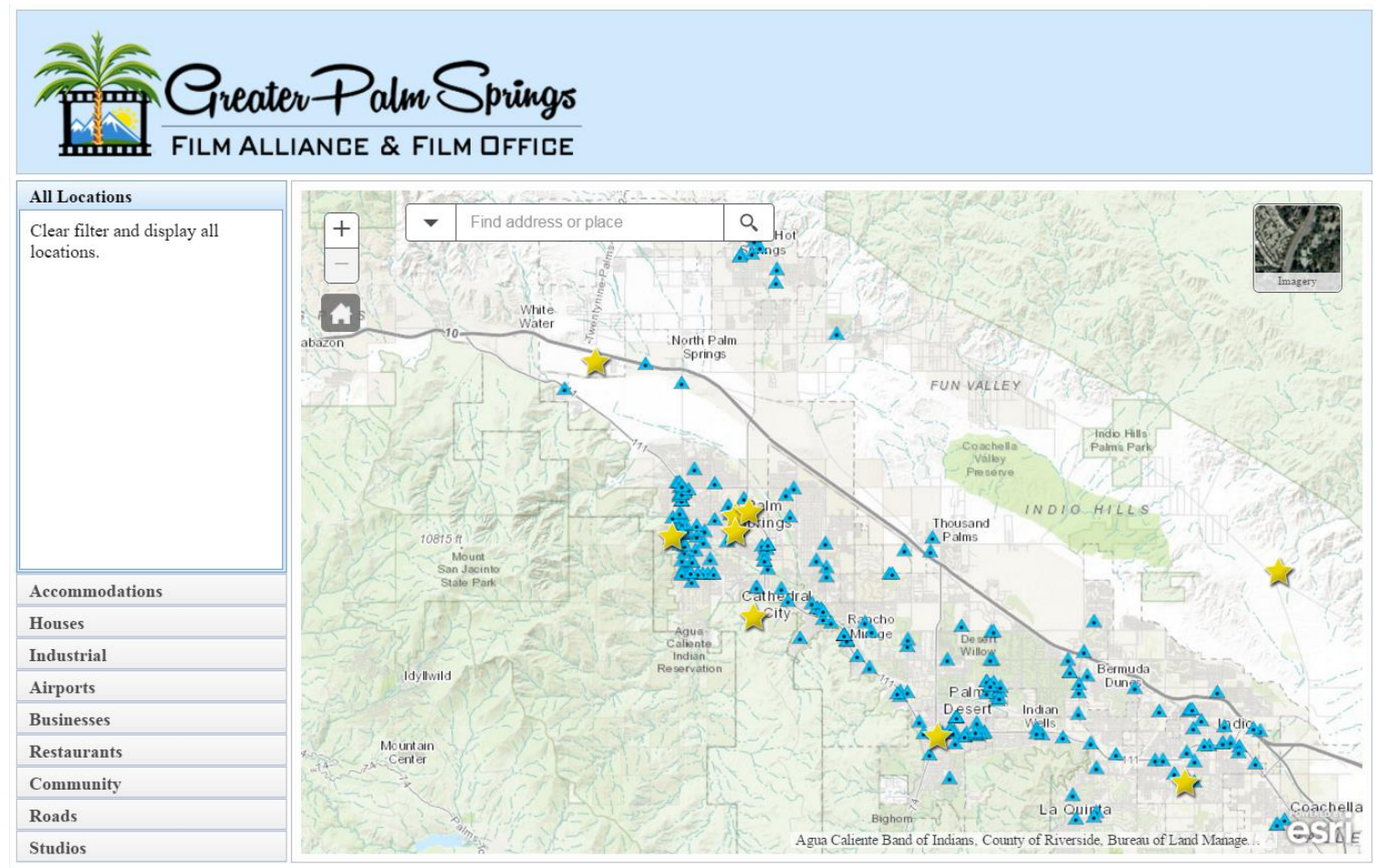

Figure 5-1: Web application user interface

A collection of Dojo layout classes-BorderContainer, AccordionContainer, and ContentPane-were used to lay out the interface. BorderContainer defines the overall region of the interface, and all other areas are defined within it. A region property associated with BorderContainer accepts text values such as top, center, right, and left to specify where the container is positioned in the interface. Additional Dojo classes were added to each of these regions to create the overall layout.

\subsubsection{Map Viewer}

In the center region of the BorderContainer class is the map viewer. Implemented as a map widget from Esri ArcGIS API 3.18 for JavaScript, the web map is programmatically created by combining an Esri basemap and the operational data from the hosted feature layers. Figure 5-2 shows the web map displaying the operational data in the center region of the BoarderContainer class. 


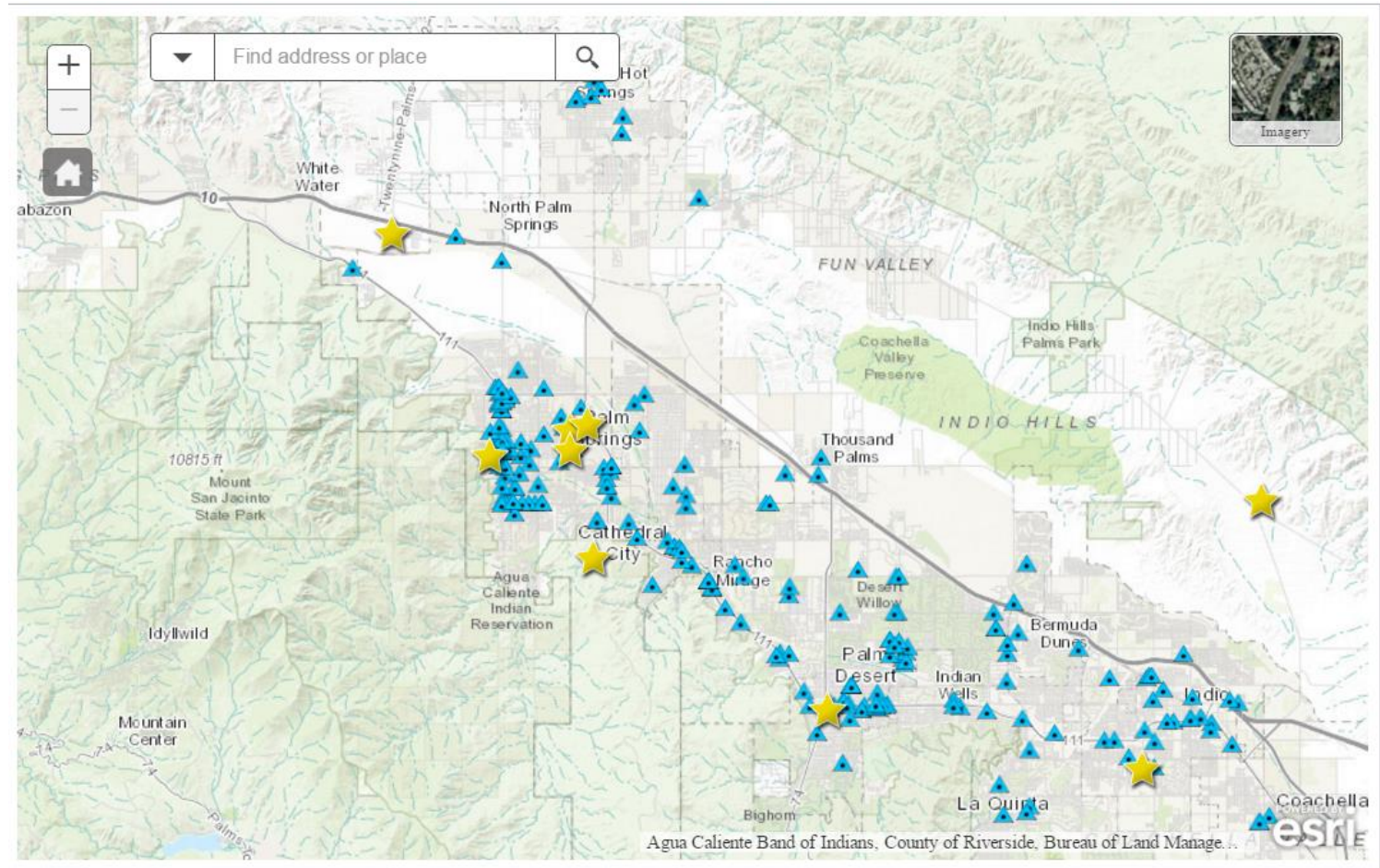

Figure 5-2: Center region of the web application user interface

The webs map was designed with standard Esri navigational and user interface widgets including the Zoom In/Out, Home, and BasemapToggle widgets. These map components are created by JavaScript code and assigned to their respective HTML <div> elements in the HTML file. The Home widget returns the web map to the map's original extent. The BasemapToggle widget was configured to allow the user to select between imagery and topographic basemaps from content in ArcGIS Online. The ArcGIS API provides many other basemaps, but for this application, the code limited the choices to two.

The Search widget was also implemented using the ArcGIS API. The configuration of the widget provides three flexible search options: search by location type, search by business type, or both. The search widget is constructed and assigned to the map and configured using several definition and configuration coding steps.

One of the first steps in configuring the search widget involves setting up the search configuration options for the search object. In the code, this setup step is called pushing. During the construction step, an empty array is assigned to the search Sources property. This purges the default search object of the World Geocoding Service locator, which is part of all search widgets by default. For this application, only search of the operational layer attributes was necessary.

Next, the search object is configured with each of the layers used for searching. The application is designed to search the Location and Vendor feature layers. The location search function is programmed to search the LocTitle, LocCat, and LocShortSum attributes. This helps to ensure the best possible chances of a successful match. As the user begins typing letters into the search box, the widget anticipates the keyword and displays recommended results based on what the user has typed. These recommended 
results are formatted in the JavaScript code to display the value from the Location Title attribute. The vendor search function is programmed to search the Name and Category attributes of the feature layer. The recommended results for a vendor search include both the business name and the vendor category for clarity. Displaying the vendor category provides additional information about the type of services provided by the business. This will also help users find the right business if they are unfamiliar with the category of vendors in the location library. When the search methods are used in combination, the search widget searches across all five attributes in the two layers.

Additional cosmetic properties are defined for the search widget, including the placeholder text displayed as a prompt for the user as well as the template for recommendations. For this project, a separate search results pop-up template was not created and the existing location and vendor pop-up templates were reused as a timesaving measure. Figure 5-3 shows the search widget in several different states of operation.

\begin{tabular}{|l|l|l|}
\hline \multicolumn{1}{|c|}{ Enter word or phrase } & Q \\
\hline \hline All & & \\
Search by Location & \\
\hline Search by Business & \\
\hline
\end{tabular}

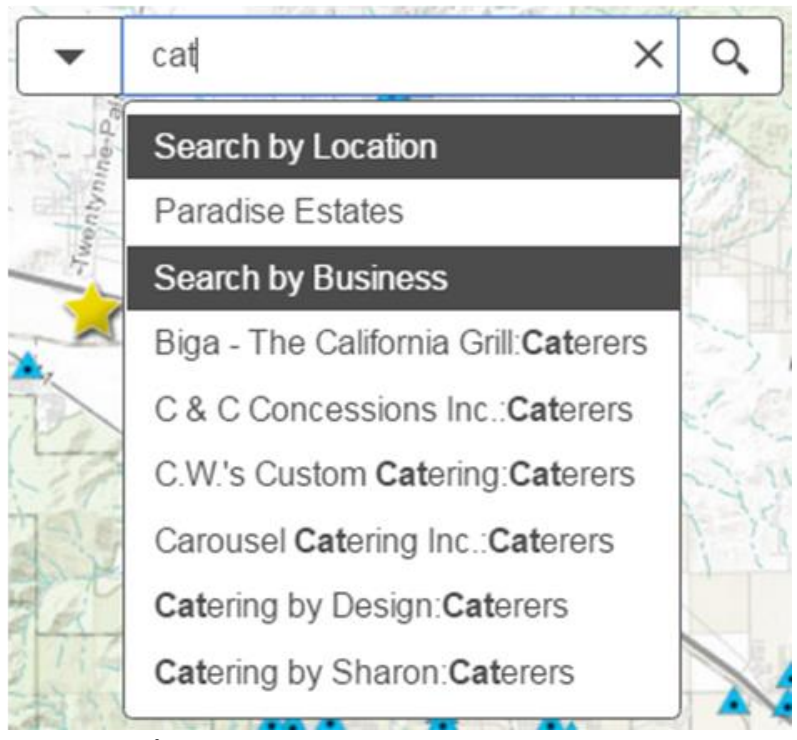

Figure 5-3: Search widget in operation

Combining the individual components of the interface creates an interactive user interface that is easy to use and puts essential search tools in the hands of the user. The application contains an extensive resource of information about locations and businesses critical to the location scout. Figure 5-4 shows the various components that make up the user interface deployed and demonstrating the usability of the web application. From this figure, one see the search widget in action, the location pop-up displaying relevant 
location information, and the filter feature activated showing only location categorizes as airport.

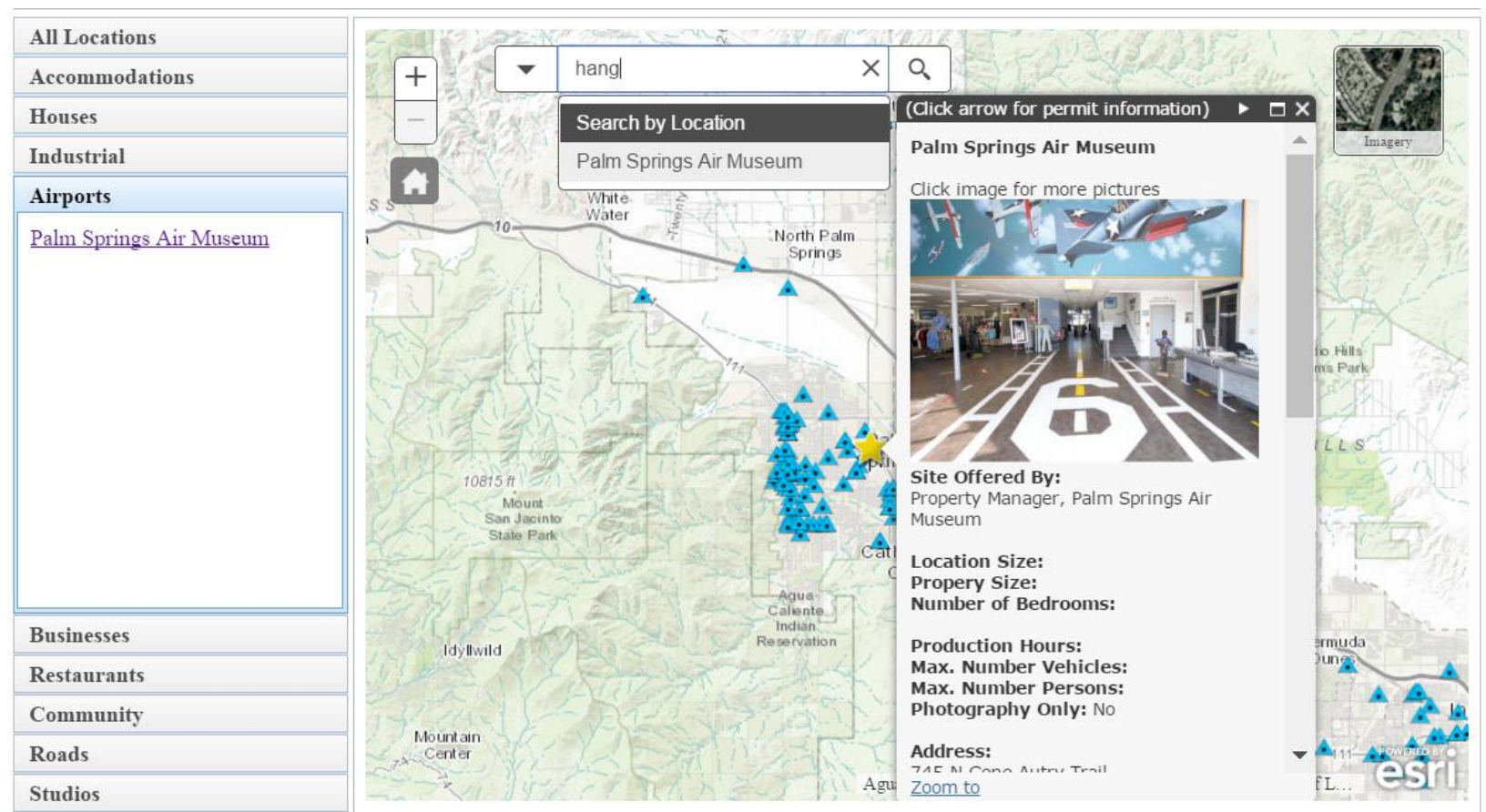

Figure 5-4: Components of the user interface working together

\subsubsection{Symbolizing Feature Services and Pop-ups}

The hosted feature layers and the resultant map service contain default symbolization. However, the decision was made to override the defaults on some feature layers by using JavaScript code in order to provide greater flexibility in the final application. While some portions of the layer symbology remain in the default state, if the client decides to make changes to the layer's symbology, the effort to do so would only involve modifying JavaScript code on the webserver and not the original feature classes. Any change to the original feature classes in the geodatabase would require the services to be republished, which would create additional issues surrounding any newly added library locations. In other words, once the client has added new locations to the library, the original feature classes and geodatabase will be out of date and should never be republished.

The Location feature layer received a custom symbol definition for displaying the map icons for each location. The Esri SimpleRenderer and SimpleMarkerSymbol classes were used to define the location markers on the Location feature layer. The SimpleMarkerSymbol class allows an image file to be used as the marker in place of a standard map symbol. In keeping with the filmmaking theme of this project, a yellow star was used to indicate locations. The SimpleMarkerSymbol class also supports customizing of the size of the marker. Figure 5-5 shows the default display of the location feature layer. All locations are displayed by default or when the All Locations category of the filter feature is selected. 


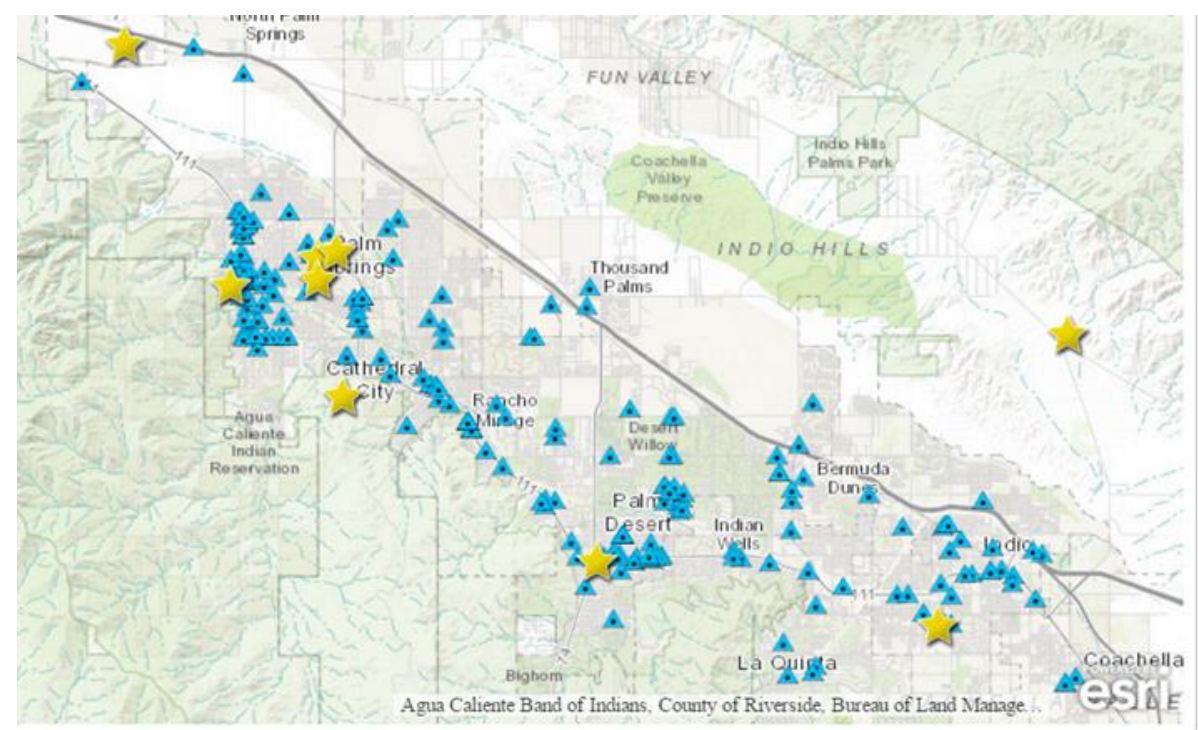

Figure 5-5: All locations in the library display by default

The second custom symbol to be defined was on the PermitDetails feature layer. By default, the polygon boundaries of each of the jurisdictions in this layer are visible. Since the actual boundaries of the jurisdictions are not required in order to present the necessary location data to the end user, the line boundaries were eliminated. Using a SimpleLineSymbol class, the polygon boundaries were suppressed by defining the line width as 0 . In both cases, the symbol definitions are applied to their respective layers by using the SimpleRenderer class.

Custom pop-up windows were defined for the location, vendor, and permit data layers. Using the Pop-upTemplate class, new templates were created in order to define which layer attributes would be displayed and how the content would be formatted. The Pop-upTemplate class extends the InfoTemplate class, providing more flexibility in defining the layout of the content. The Pop-upTemplate can accept any content that is formatted using HTML.

Since location data and photos are the cornerstone of this project, significant effort was expended on defining the Location feature layer pop-up window and ensuring that the data were easily accessible. Nearly every attribute from the Location feature layer is displayed in the window. Using HTML, the attribute values were customized in an easyto-read format that puts important information at the top of the window. A thumbnail image is displayed for each location. By using the HTML tag 〈a href>, the thumbnail is also a hyperlink to additional photos when available.

Also, PermitDetails feature layer contains important decision-making information that should be presented in the early stages of the scouting processes. Because of this fact, permit data appears along with the location data. This approach presents location and permit data as soon as a location icon is selected. The PermitData feature layer popup window was created using a technique that was similar to the Location feature layer pop-up, using HTML to format most of the feature layer attributes in an easy-to-read format. One of the permit layer attributes stores a uniform resource locator (URL) value that references a website. This hyperlink redirects the user's browser to the governing jurisdiction's website for additional details about applying for filming permits. If the 
agency does not maintain such information on the web, the main website URL is stored instead. Most importantly, the contact name, phone number, and email information are provided when supplied so that the user could contact the agency for additional permit details.

The vendor pop-up definition follows a similar workflow but contains only basic business and contact information.

\subsubsection{Content Pane and Filter Feature}

The main user interface of the application uses the Dojo BorderContainer class to define several regions where additional components are instantiated. One important region, identified as leftPane, was further defined using the Dojo AccordionContainer class. This region was subdivided into several Dojo ContainerPane classes. This collection of panes within the overall AccordionContainer class makes up the location filter feature of the application. The location filter feature allows the user to select a location category of interest so that the map will update to display only those locations identified by the selected category. Figure 5-6 shows the location filter feature presented in the Dojo Toolkit AccordionContainer class.

\begin{tabular}{|l|}
\hline All Locations \\
\hline Accommodations \\
\hline Houses \\
\hline Industrial \\
\hline Airports \\
\hline Businesses \\
\hline Restaurants \\
\hline Community \\
\hline Roads \\
\hline Berdoo Canyon Road \\
\hline Kellogg Road \& Pipline Road \\
\hline \hline Studios \\
\hline
\end{tabular}

Figure 5-6: Left region of the user interface showing the location filter feature

At the time that each of the filter feature content panes is created, the JavaScript code assigns several properties to each element that are essential to the operation of the feature. The filter feature operates by applying a new definition query to the location feature layer each time the user selects a content pane. Within each content pane, location titles are displayed for only those locations assigned within the selected category. The 
definition query requires the integer value that represents the location category, rather than the text description; this is in order for the definition query to function on the location feature layer. One challenge that arose during implementation was that when querying locations, it is the integer value that is returned and not the category description. While this does not pose a problem for constructing the definition query, it does when creating the category titles on each of the panes. Since the integer values are numeric codes, they do not make effective human-readable titles for the filter panes, and additional work was necessary. To overcome this obstacle, a cross-reference data structure between the location category integer and the text description had to be created. The cross-reference was implemented by executing a separate query on the LocationCategory table in the feature service. The results of this query were then formatted into a dictionary data structure, with the integer value as the key and the description as the value. Using this dictionary allows the code to programmatically add category titles to each of the content panes for the filter feature.

The filter feature is created using the ContentPane class. Figure 5-7 is a representation of the ContentPane creation workflow.

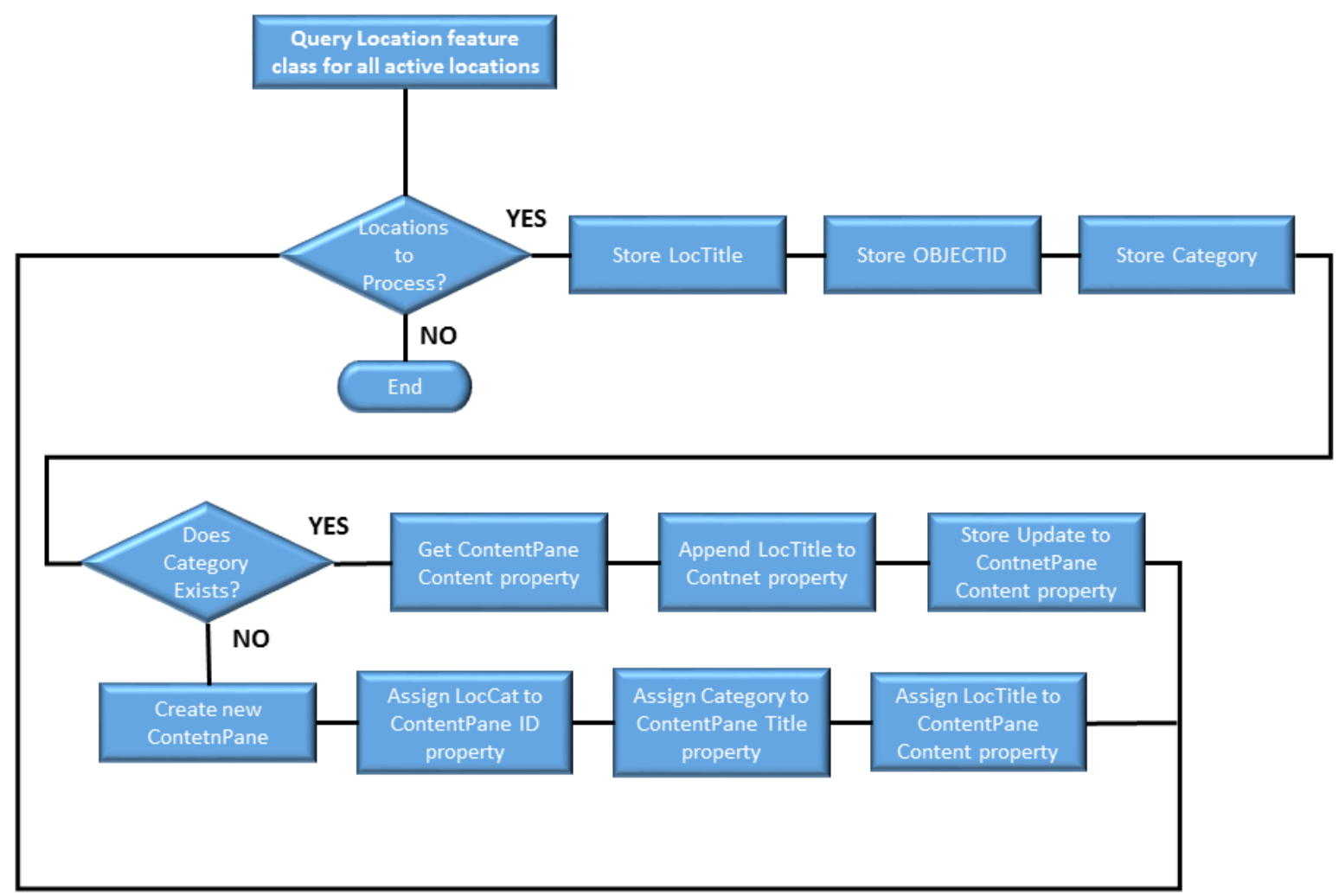

\section{Figure 5-7: Location filter feature and ContentPane creation workflow}

Once the location category description, integer value, and location titles are available, the creation of the individual content panes may continue. Looping through all locations returned from the location query, the code checks to determine whether the 
current location category has already been assigned to a content pane. If not, a new content pane is created; assigned an ID property equivalent to the category integer value; and, using the cross-reference dictionary, given a title property equivalent to the category description. If the category already exists, the code retrieves the current content for that pane and concatenates additional data with it based on the location currently being processed in the loop. This ensures that if there are multiple locations in the result set that are classified with the same category, a duplicate pane is not created and that the pane's content is updated to add the new location to it. Concatenation is used so that previously added location titles are not overwritten. As part of this process, the category integer value and location titles are obtained from the result set, while the category description used for the content pane title and definition query is obtained from the cross-reference data structure.

The final piece of the location filter feature is the event listener, which applies the definition query. A JavaScript event listener is set up on the selectedChildWidget event of the leftPane element. When a user selects a category of interest, the selectedChildWidget event fires. A function executes and evaluates the value of the pane's ID property that contains the category integer value. If the value is 999 , the definition query is removed, and the map returns to the default display of all locations. Otherwise, the definition query is updated using the integer value from the pane ID property and applied to the layer using the setDefinitionExpression method to update the map.

\subsection{Summary}

The implementation of the project involved three Esri GIS technologies: ArcGIS for Desktop, ArcGIS Online, and ArcGIS API for JavaScript. The ArcGIS for Desktop suite was used for creating and managing the data and file geodatabase prior to publishing. Since ArcGIS Online was to be the final hosting location for the operational and reference data, a hosted feature service was published to ArcGIS Online from the file geodatabase. In addition, since a web application was to be the final product, ArcGIS API for JavaScript was used to develop the custom application. Web development techniques incorporating HTML, CSS, JavaScript, the ArcGIS API, and Dojo Toolkit were used to design and implement the web application. 



\section{Chapter 6 - Use Cases}

One of the intended goals of this project was to streamline the client's location scouting process to make the job easier. By deploying a self-service scouting tool, the client anticipates being able to serve a much broader audience of film and television professionals searching for appropriate production locations. The online location library was designed to support several different production workflows including location scouts, partner film commission members, and the client himself. The following sections present each of these three use cases in more detail.

\subsection{Use Case: Location Scouts}

One of the challenges associated with the client's current process has been the sheer number of inquiries received at the film office. With limited budget and staff, the Greater Palm Springs Film Alliance (GPSFA) faces increasing challenges in adequately responding to location requests. Fueled by the growing popularity of the Coachella Valley as a tourist destination, more and more film and television productions are seeking out the iconic landmarks and signature architecture found in the region. Enabling faster response to inquiries would allow the client to handle the increasing number of requests coming into the office. The production staff responsible for finding a location for a film or television shoot often has only a description of locations for a project. This might include an architectural style, like mid-century modern or Mediterranean, but may also include specific setting descriptions, like airport hangar or desert road. The online location library would streamline this search process and put the site inventory into the hands of every location scout with access to a web browser.

For example, an airport hangar is needed. A location scout accesses the online location library via a supported web browser. The scout begins typing the keyword "hangar" into the search box and gets immediate recommendations from among the locations in the library that match that word. Figure 6-1 shows an example of a location of a partial keyword match. A user begins typing the keyword "hangar" and before completing the word, the search functionality recommends possible matches from the library as the user types. 


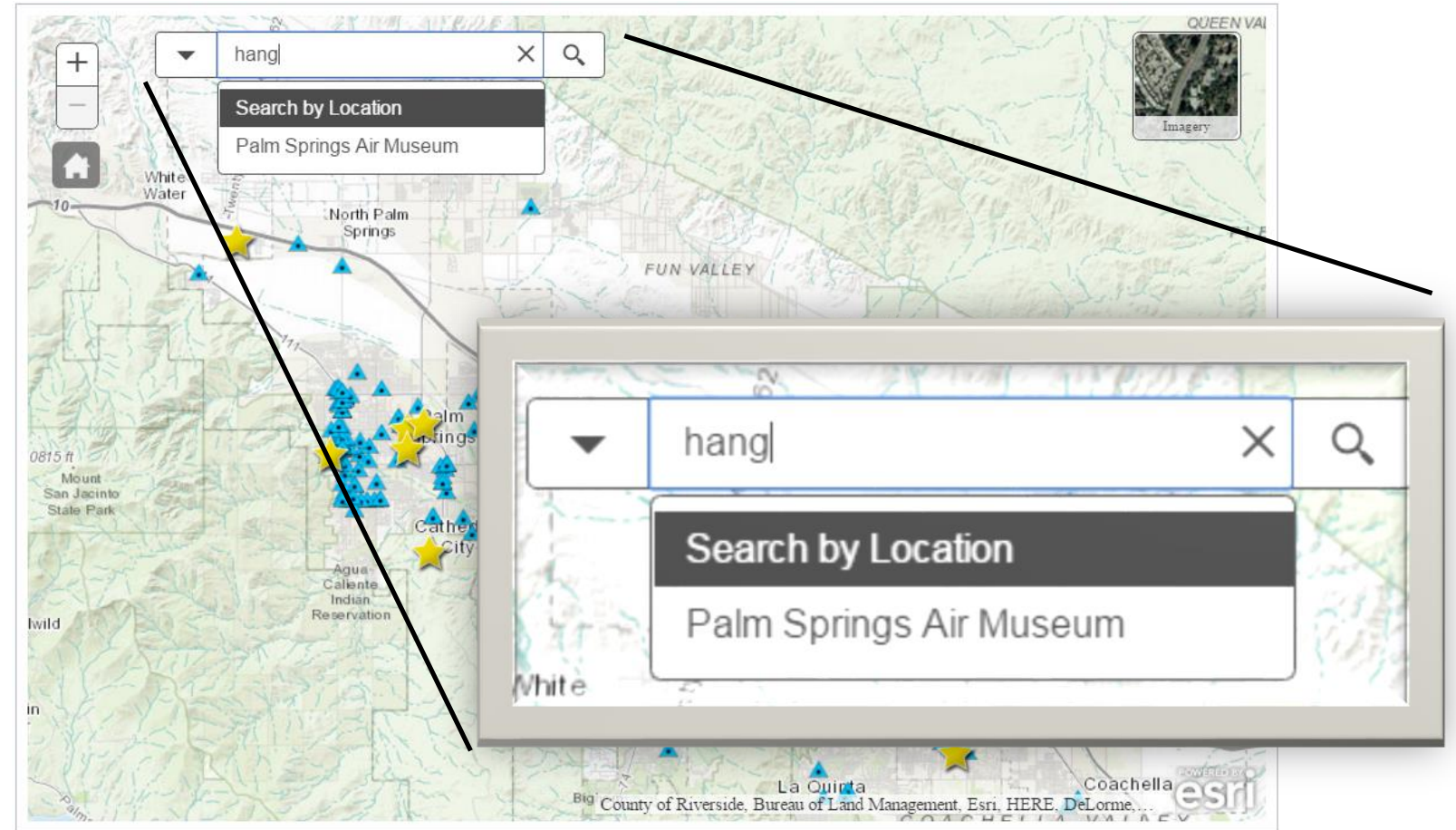

Figure 6-1: Search library by location type

Once a match is made, the location scout selects the result of interest, and the map automatically zooms to the selected location and displays the location pop-up, as shown in Figure 6.2. This pop-up window provides detailed information about the site including location title (e.g., Palm Springs Air Museum or Paradise Estates), address, point of contact, and relevant scheduling details such as the hours the location is available, or the maximum number of people and vehicles allowed on-site. 


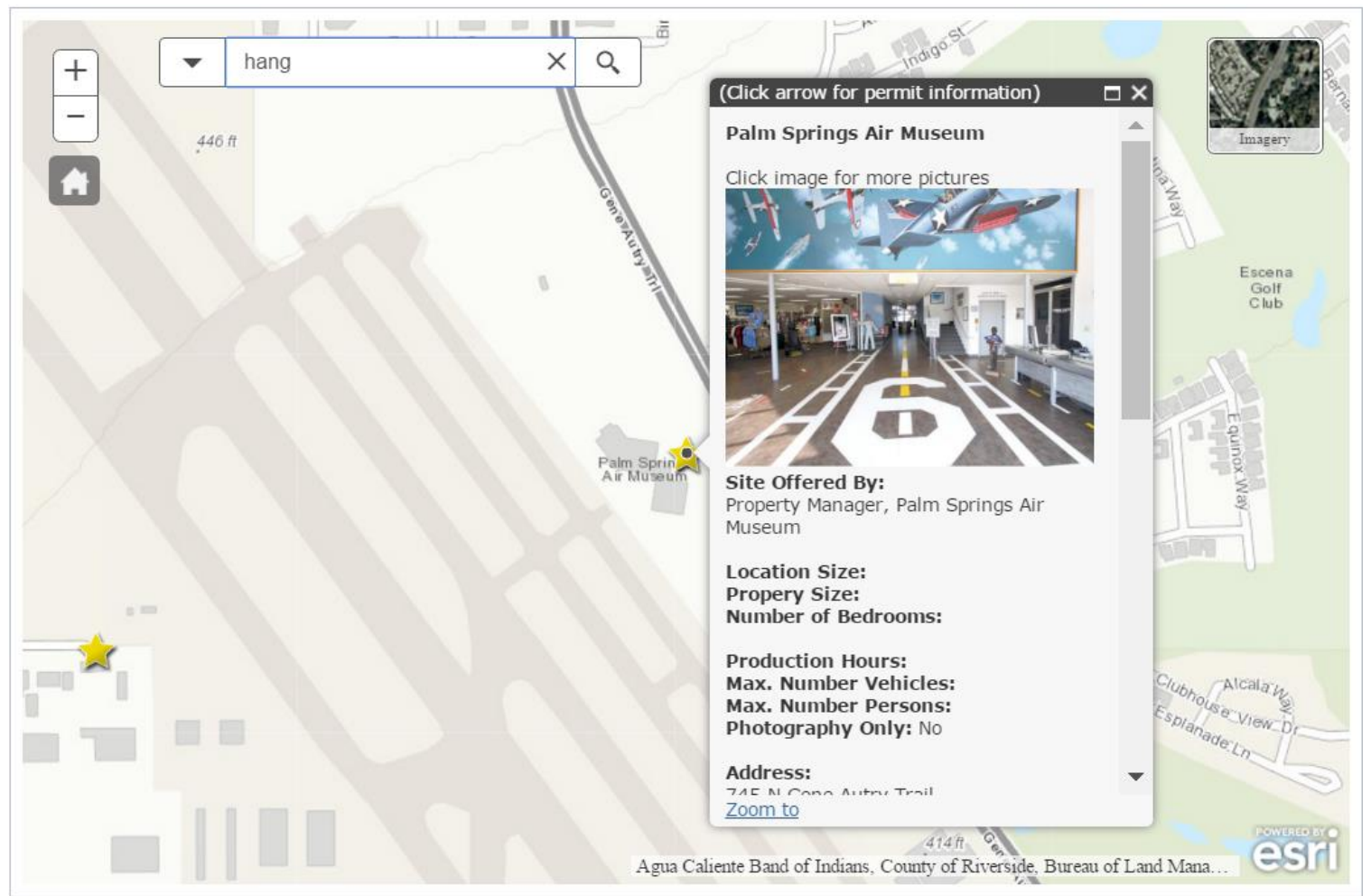

Figure 6-2: Location pop-up displaying relevant site and production information

Almost immediately, the scout has the necessary information to make a decision as to whether the site is viable. Moreover, this all happens without having to make direct contact with the film alliance office or having to wait for staff to check location inventory lists.

Another feature of the online location library that would benefit the location scout is the extensive database of local businesses and service providers that support production activities. This business information is accessible from the same interface as locations and displayed on the same map. After finding location matches, the scout may proceed to explore the map for local vendors and service providers. Figure 6-3 shows the map interface indicating both locations and vendors along with the vendor pop-up window, which displays contact information for the selected business. 


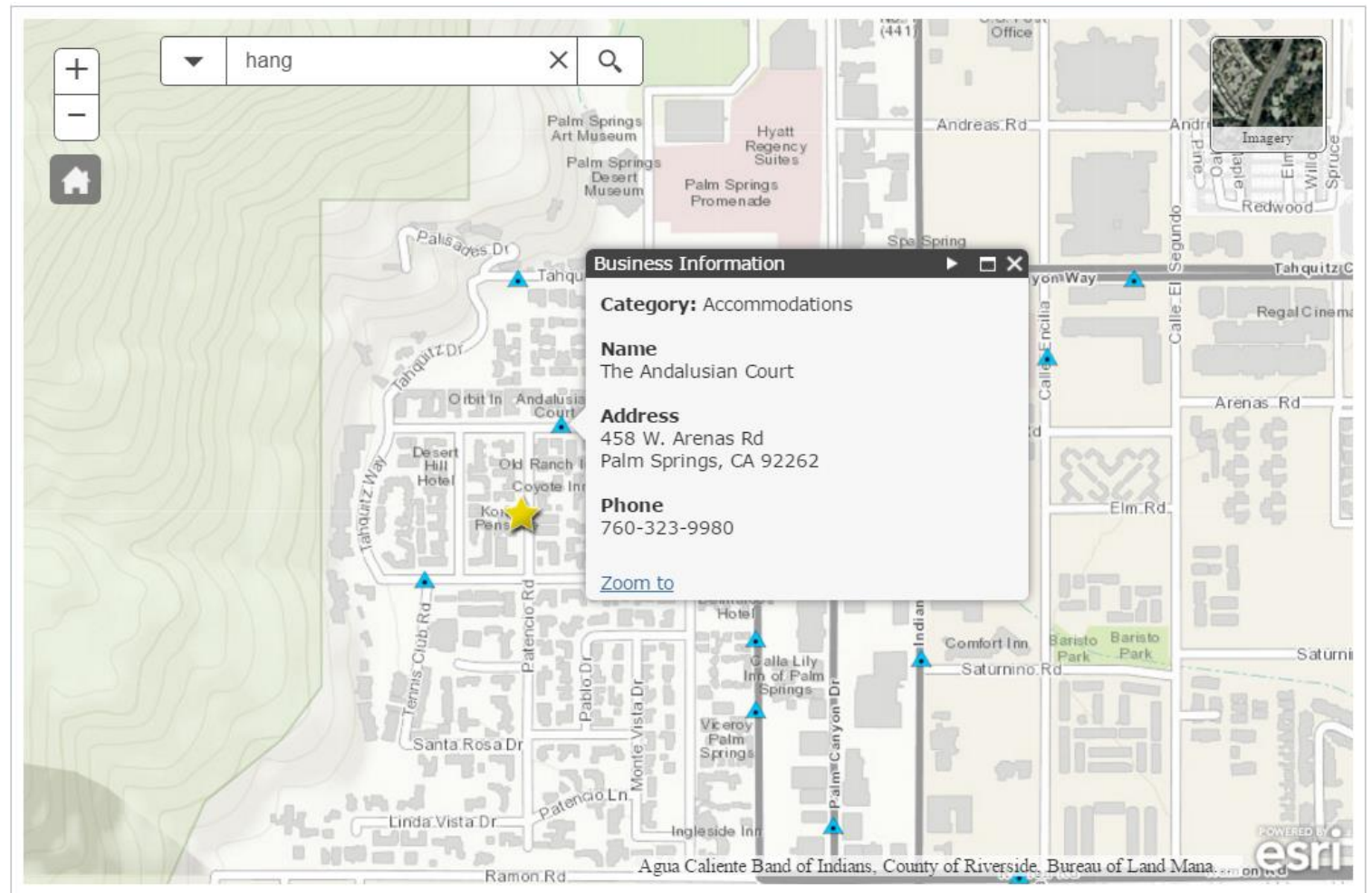

Figure 6-3: Application interface showing local business information

One other method for a location scout to find matching locations is to use the interactive filter feature of the application. If a production needs locations of a particular category, the location scout can use the filter pane, on the left of the user interface, to filter the map and display only those locations identified with the particular category. Figure 6-4 shows the category Roads selected in the filter feature as well as the map displaying two locations of that category, together with icons representing local filmfriendly businesses. 


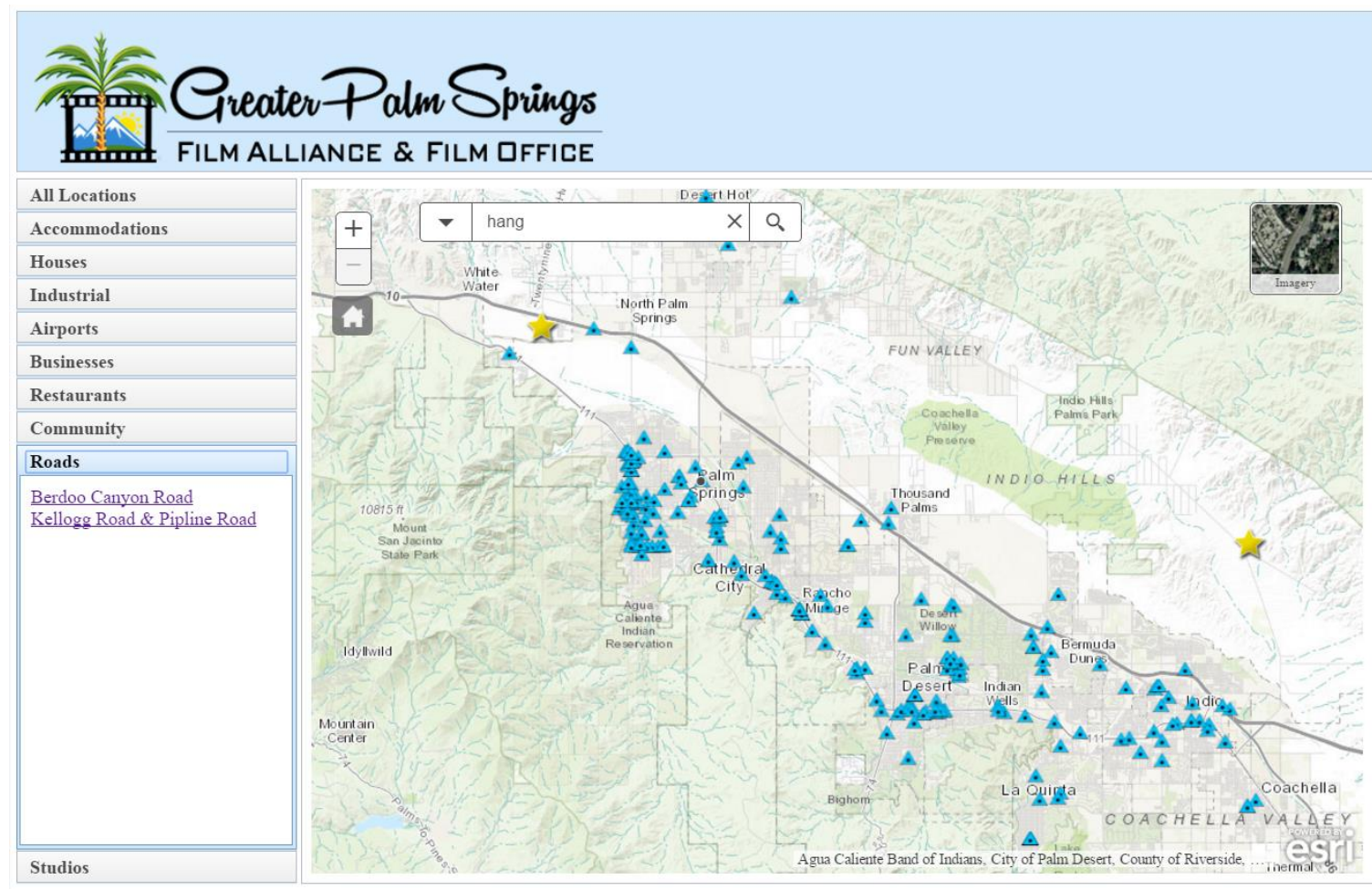

Figure 6-4: Location filter feature showing only one category

This web application combined several different data sources. The goal in consolidating sources was to put all this information into one location for ease of use. A dataset containing film permitting requirements is part of the online location library. Figure 6-5 shows the Filming Permit Information pop-up displaying permit application details for the local jurisdiction. Filming permit details are accessed from the location details display by clicking the right arrow. The user may return to the location information by clicking the left arrow on the permit information display. 


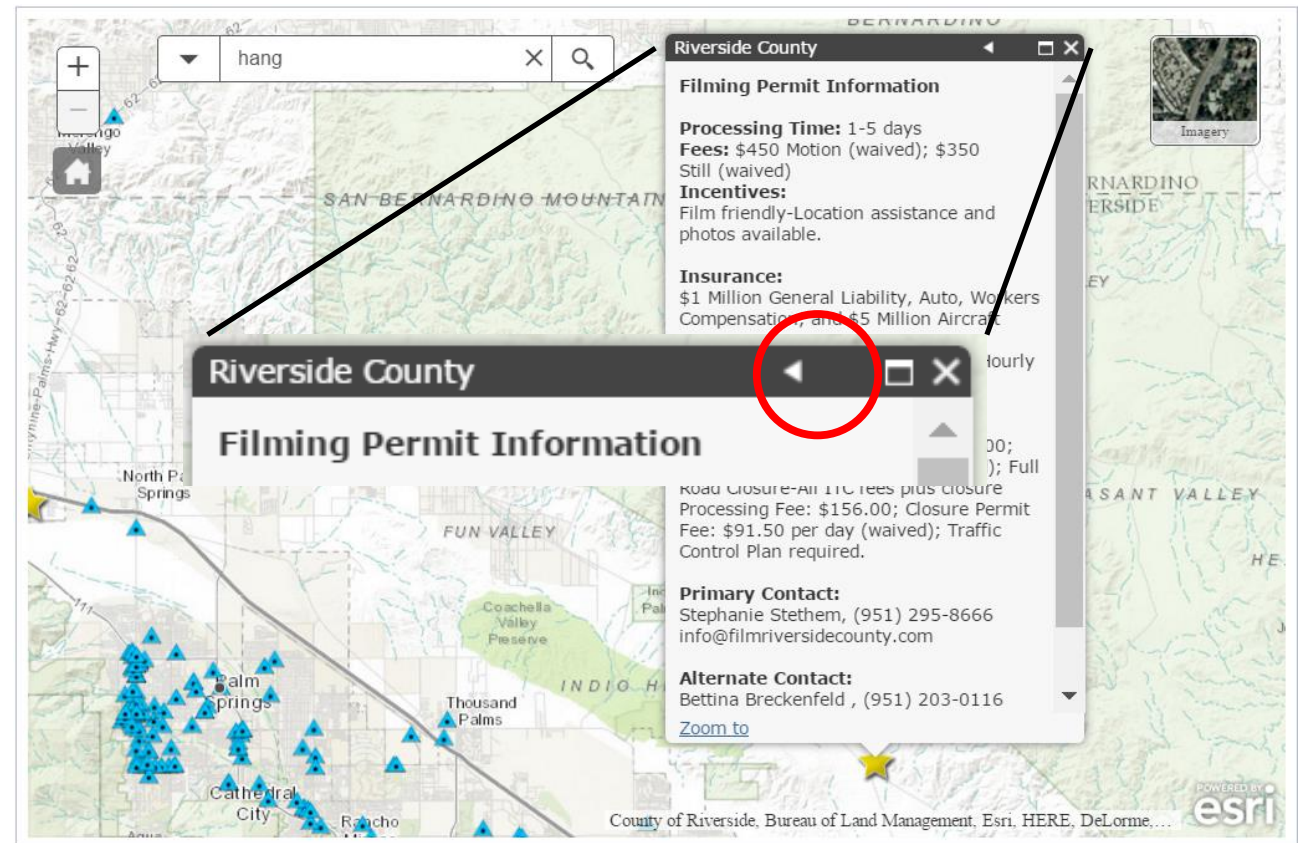

Figure 6-5: Film permit details for Riverside County

Bringing the location, vendor, and permit information into a single online source will streamline the scouting process. Presented in the same pop-up window as location details, the permit details for the agency with jurisdiction over the location are critical to the production planning phase. With some production timelines being very tight, the location scout needs to know about permit processing time and fees as part of determining the viability of a particular site. If the permit application process would exceed the production schedule, that particular location would not be considered viable. An ancillary outcome of the project is that location scouts and other film and television professionals could use this same web application when planning a production schedule. By understanding the processing time for film permits in advance, it can be built into the production schedule to set reasonable expectations and produce a more accurate timeline.

\subsection{Use Case: Partner Film Commission Members}

A second use case addresses the workflow for a member of a partner film commission or film office. As part of a typical location scouting process, one film office may contacts while searching for a site. Having an online location library accessible from any supported web browser would make this process easier and eliminate slowdowns due to lack of coordination between offices.

When a partner film office receives a location request it is unable to fulfill, other film offices may have a suitable location in their inventory. It is quite common for regional film offices and commissions to work together to ensure that the production books a location in their area. Partner members provide support to one another through direct communication, which can slow down the scouting processes. When one film office has its location inventory online in a searchable database, staff from other film offices have the same ability to access the library and search. Moreover, by reducing the amount of direct coordination necessary between film office staff member, the time to produce a list 
of viable location matches may be shortened. Reducing the turnaround time for location requests benefits the location scout, the film office, and the local communities they support.

The workflow for the partner commission member is essentially the same as for a location scout. The commission member would access the web application via browser and search the inventory based on location category or other descriptors. Once a match has been identified, the commission member would have access to all the relevant information necessary for the location scout to make a decision.

\subsection{Use Case: The Client}

The third use case covers the workflow for the client himself. While the design of the online location library was driven primarily by the needs of the client to enable location scout self-service, the client himself stands to benefit from the easily accessible location library. Additionally, this online location library enables a new workflow for an authorized administrator for adding locations to the library but would not be part of the other use cases.

The client would use the same workflow as a location scout or partner commission member. Location inventory is searched using keywords via the web-based application. Results from the search are displayed on the map, along with production details. The client may choose this route rather than referring a prospect to the web application, especially for inquiries by high-value or large production clients that require extra expertise.

This project's client has one additional workflow not shared by the location scout or partner commission member, and that workflow is for adding locations. When the client acquires or otherwise identifies new locations throughout the study area, he needs the capability to add the entry into the database. This process involves geolocating the new site on the map and entering all the relevant information about it, including the production details and contact information. Currently, this process takes place outside the location library web interface. New locations are added to the database using ArcGIS Online and the hosted web map. Figure 6-6 shows the ArcGIS Online interface for adding a location to the library. 


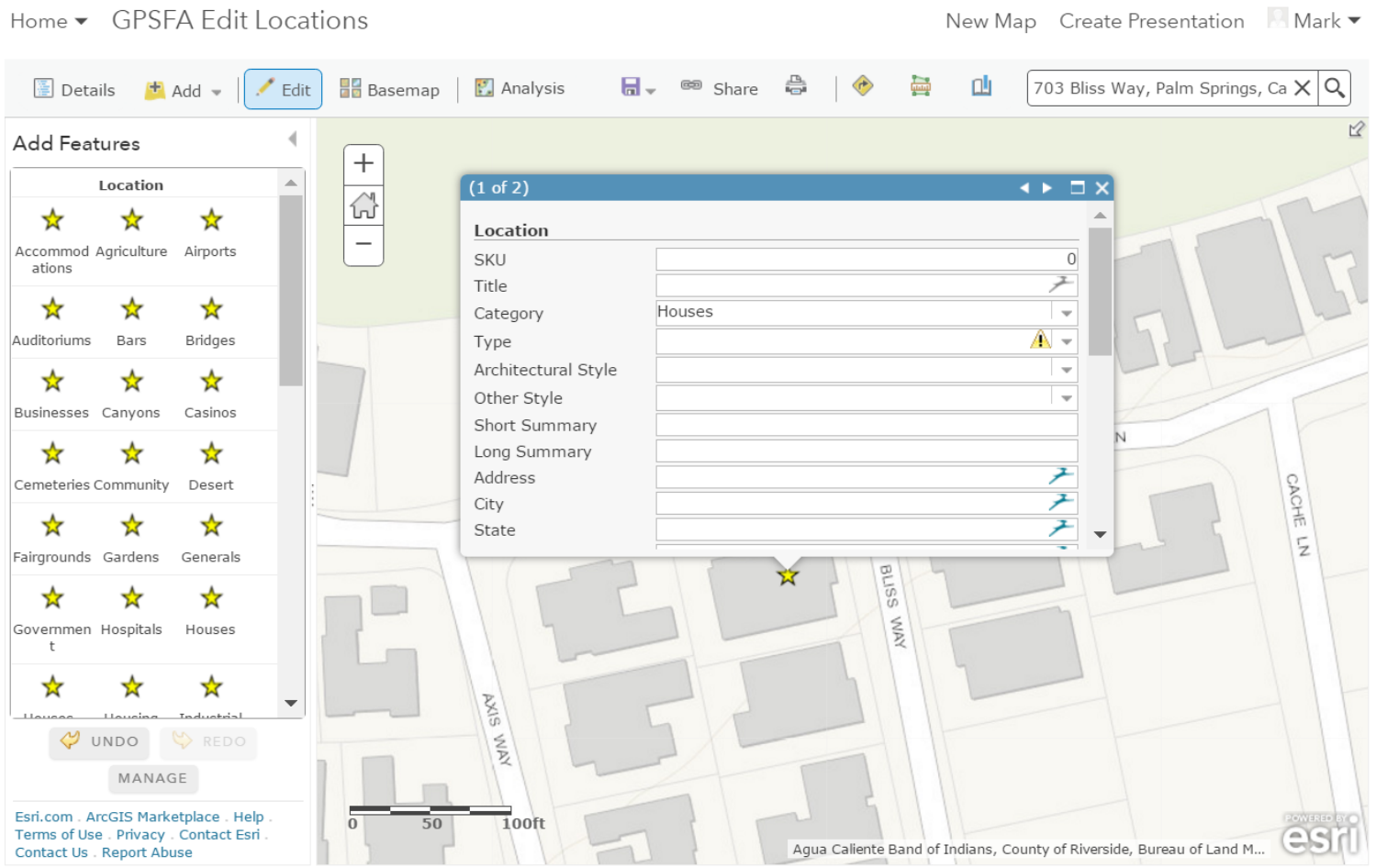

Figure 6-6: Administrative interface for adding locations

This workflow involves accessing the web map via ArcGIS Online with an authorized ArcGIS Online organizational account. The user clicks the Edit menu item to put the map into editing mode, selects the appropriate Add Feature template, and clicks the map at the site of the new location. The map interface has a search widget that allows the editor to search for an address to find a location. The data entry window appears, and the administrator completes all the required and optional fields for each new location and closes the data entry form. Clicking the Edit button returns the map to viewing mode.

\subsection{Summary}

Chapter 6 provided detailed explanations of the three main use cases addressed by the application. While each use cases stands alone, the workflows for interacting with the online location library are similar to each other. The basic workflow involves searching for a location of interest based on a keyword search of location attributes. Common keywords are types of architectural styles or buildings of interest.

This project introduced an additional workflow for the client. This workflow involves an authorized user adding new locations or updating existing ones. This process does not take place in the web application user interface. Instead, adding or editing locations is performed in the ArcGIS Online interface via the GPSFA Edit Locations web map. This map is only accessible to authorized users with a valid ArcGIS Online organizational account. 


\section{Chapter 7 - Conclusions and Future Work}

At the end of any project, it is often helpful to the implementation and development team members to reflect on the process from beginning to end. The purpose of such reflection is threefold. First, analyzing the entire project-from conception and definition to implementation and project close - can help them determine where the project succeeded and where areas of improvement may exist. Second, reflection on an effort of this magnitude provides an excellent opportunity for team members to add lessons learned to their body of knowledge for future projects. Lastly, the project team can use this time to appreciate the level of commitment and dedication necessary in order to understand the client's needs and translate them into a viable technology solution. To that end, Section 7.1 reviews and summarizes the activities of this project, in search of those lessons that will inform and improve future projects. Section 7.2 uses the information gathered from Section 7.1 to make intelligent recommendations of possible future work or enhancements to this project.

\subsection{Project Summary}

The primary objective of this project was to make the client's job of film and television production location scouting easier by using technology to streamline the workflow. Project requirements were defined in support of the primary objective. These included displaying locations and pictures on a map; consolidating multiple data sources; and creating an interactive, web-based tool.

A secondary objective of the project was to help minimize the likelihood of runaway productions - films and television shows that are conceived in California but produced elsewhere - and which have well documented negative economic impacts on the state's economy. The project combined web-development technologies with a geographic information system (GIS) to create an online location library. The web application is easy enough for the least tech-savvy industry professional to use. Its self-service design accomplishes multiple goals for different end users. The steps of the development process are discussed in this section.

After gathering business requirements and developing an understanding of the business of location scouting, the project team settled on designing a web application built using HTML, JavaScript, and ArcGIS API for JavaScript. The early stages of the implementation involved developing an Esri file geodatabase and loading the client's datasets into the ArcGIS for Desktop. The location, person, and vendor data provided by the client were received in Excel format and required review, cleaning, and geocoding before they could be used. Once all data were loaded into ArcGIS for Desktop, an array of geodatabase features were used to represent the real-world locations and relationships including geodatabase domains, subtypes, and relationship classes.

Since the outcome of this project was to be a web-based application, the geodatabase - including all feature classes, relationship classes, and nonspatial tableswas published as a hosted feature service in the ArcGIS Online environment. This allowed the majority of the file geodatabase features and functions to be available online and in the subsequent web map. Publishing the data as a hosted feature service created 
ArcGIS Online feature layers for each of the data layers. These features services exist in Representational State Transfer (REST) format and are accessible via JavaScript.

Once all of the client data were properly formatted and published in an online format, the work of programming the web application took place. Using web development tools including HTML5, Cascading Style Sheets (CSS), JavaScript, and ArcGIS API for JavaScript, a custom application was created. The development involved extensive use of the tools and widgets that are part of the ArcGIS API. An interactive interface was designed using Dojo Toolkit and presented a web map displaying the client data from the hosted feature layers. Standard map tools were programmed into the interface and included a custom widget for searching locations and businesses by matching keywords. A custom location filter feature was created to interactively filter the map display based on a user-selected location category.

The core functional requirements agreed upon by the client and project team were successfully delivered. The web application presents the client's location data and site pictures on a map; data from several different websites, spreadsheets, and other sources were consolidated into three hosted feature layers and accessible from a single interface; and the application is web-based. The development process used industry-standard tools and techniques, and the web application was successfully tested in both the Google Chrome and Microsoft Internet Explorer web browsers. Since the pilot application was implemented using a small sample dataset, performance of the application on the client's existing webserver cannot be reliably tested until the database contains substantially more data.

\subsection{Future Work}

This project focused on addressing the client's current challenges to the location scouting processes. Critically needed functions were part of the original scope of the project. Unfortunately, there were other functions that could not be considered part of the scope. This section reviews a sample of features and enhancements that may be considered by the client in the future.

The current design of the pilot application allows the end user to manually explore businesses and service providers in the vicinity. One improvement would be to add the capability for the end user to perform a proximity search for businesses within a certain radius of the location of interest. This function could be further enhanced by allowing the user to use a drop-down menu to select pre-defined distances for the proximity search. Additionally, analyzing the drive time from the location to a business would be helpful when production planners are considering how to move people and equipment in and out of a particular location. In addition, enabling the proximity search to be limited to businesses of a particular type would be useful.

The current application allows the map to be filtered by location category. The end user selects the category of interest, and the map updates to display only those locations assigned that category. A similar filter feature for businesses would be useful for the end user. This function would allow the location scout to focus on particular businesses of interest when assessing the viability of a location.

At this time, an authorized administrator is the only user capable of adding new locations or editing existing ones. Developing an easy-to-use interface that would enable anyone to add new locations would allow the location library to grow much more 
quickly. There would need to be a quality assurance/quality control (QA/QC) process in place as well to prevent bad data from getting into the library. The data schema for the pilot project was intentionally built with an attribute to help facilitate the QA/QC process. 



\section{Works Cited}

Anglo-American Film Deadlock Broken by Pact. (1948, March 12). The Spartanburg Herald, p. 1. Retrieved from https://news.google.com/newspapers?id=UVcsAAAAIBAJ\&sjid=HssEAAAAIBAJ $\& p g=3547,1241931$

Battista, P. (2014). "Runaway" Film and Television Production: Carrots, Sticks, \& International Tax Reform. Hastings Communications and Entertainment Law Journal, 36(2), 243-305.

Business Transportation and Housing Agency. California Film and Television Incentive Program (2009).

California Film Commission. (n.d.). California Regional Film Partnership page. Retrieved February 27, 2016, from http://www.film.ca.gov/

Coachella Valley Economic Partnership. (n.d.). About Page. Retrieved March 3, 2016, from http://www.cvep.com/about.html

Cucco, M., \& Richeri, G. (2011). Film Commissions as a Driver for Economic and Cultural Development. Fourth Euro-Mediterranean Dialogue on Public Management. Retrieved from http://www.med-eu.org/

Dojo Toolkit. (n.d.). Retrieved November 30, 2016, from https://dojotoolkit.org/

Esri Inc. (n.d.-a). API Reference | ArcGIS API for JavaScript 3.18. Retrieved November 30, 2016, from https://developers.arcgis.com/javascript/3/jsapi/

Esri Inc. (n.d.-b). Samples | ArcGIS API for JavaScript 3.18. Retrieved November 30, 2016, from https://developers.arcgis.com/javascript/3/jssamples/

Fu, P., \& Sun, J. (2011). Web GIS: principles and applications (First). Redlands: Esri Press.

Greater Palm Springs Film Alliance. (n.d.). About page. Retrieved February 27, 2016, from http://palmspringsfilm.com/about/

Hozic, A. (2001). Hollyworld. Ithaca, United States: Cornell University Press.

Johnson-Yale, C. (2015). Frozen in Hollywood : Postwar Film Policy and the New Power-Geometry of Globalizing Production Labor, 32(1), 33-47.

Klowden, K., Chatterjee, A., \& Hynek, C. F. (2010). Film Flight : Lost Production and Its Economic Impact on California.

Palm Springs Bureau of Tourism. (2016). Hollywood's Playground - Official Palm Springs, California Tourism Website. Retrieved March 3, 2016, from http://www.visitpalmsprings.com/page/hollywood-s-playground/8183

U.S. Department of Commerce. (2000). US Losses from Runaway Film Production. Retrieved from http://www.ita.doc.gov/media/filmreport.html

Vincent, L. (2016). Personal Communication.

Yale, C. K. (2010). Runaway Film Production: A Critical History Of Hollywood's 
Outsourcing Discourse. Retrieved from

https://www.ideals.illinois.edu/handle/2142/18481 


\section{Appendix A. HTML Source Code}

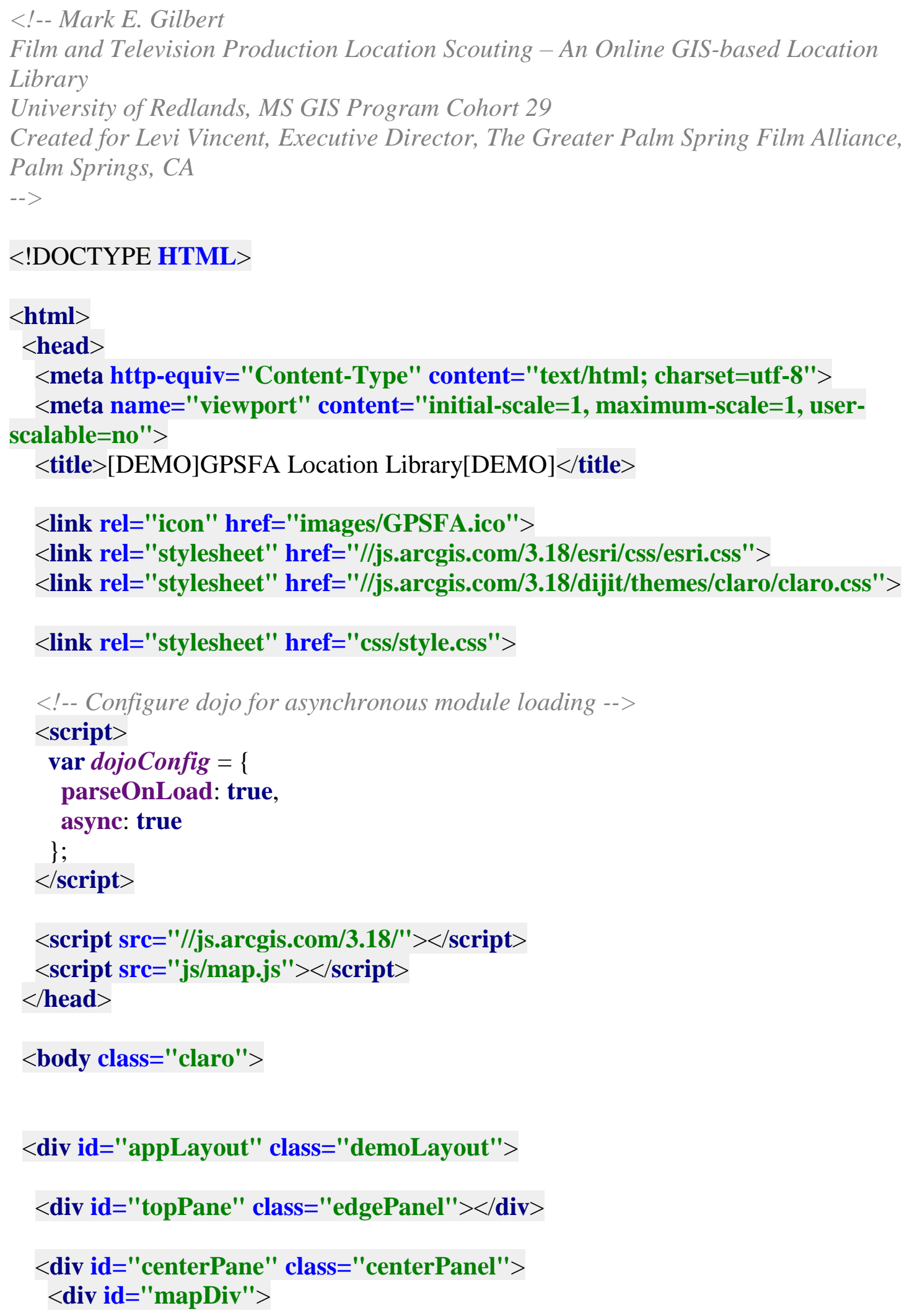




\author{
$\langle$ div id="pop-up" $\rangle\langle/$ div $>$ \\ $</$ div $>$ \\ $\langle$ div id="HomeButton" $\rangle\langle/$ div $\rangle$ \\ $<$ div id="baseMapToggle" $></$ div $>$ \\ $\langle$ div id="search" $></$ div $>$ \\ $</$ div $>$ \\ $\langle$ div id="leftCol" class="edgePanel" $\rangle\langle$ div $\rangle$ \\ $</$ div $>$ \\ $</$ body $>$ \\ $</$ html $>$
}




\title{
Appendix B. JavaScript Source Code
}

\author{
var map; \\ var leftPane; \\ require([ \\ "esri/map", \\ "esri/layers/FeatureLayer", \\ "esri/renderers/SimpleRenderer", \\ "esri/symbols/PictureMarkerSymbol", \\ "esri/symbols/SimpleLineSymbol", \\ "esri/InfoTemplate", \\ "esri/dijit/Pop-up", \\ "esri/dijit/Pop-upTemplate", \\ "esri/dijit/Search", \\ "esri/dijit/HomeButton", \\ "esri/dijit/BasemapToggle", \\ "esri/geometry/Extent", \\ "esri/SpatialReference", \\ "esri/geometry/webMercatorUtils", \\ "esri/tasks/QueryTask", \\ "esri/tasks/query", \\ "dijit/layout/BorderContainer", \\ "dijit/layout/AccordionContainer", \\ "dijit/layout/ContentPane", \\ "dojo/_base/json", \\ "dojo/domReady!"],

\section{function(} \\ Map, \\ FeatureLayer, \\ SimpleRenderer, \\ PictureMarkerSymbol, \\ SimpleLineSymbol, \\ InfoTemplate, \\ Pop-up, \\ Pop-upTemplate, \\ Search, \\ HomeButton, \\ BasemapToggle, \\ Extent, \\ SpatialReference, \\ webMercatorUtils,
}


QueryTask,

Query,

BorderContainer,

AccordionContainer,

ContentPane,

dojo) \{

//Some code adapted from sample code preseted by Esri at https://developers.arcgis.com/javascript/3/jssamples/

//and presented in the ArcGIS API for JavaScript at https://developers.arcgis.com/javascript/3/jsapi/

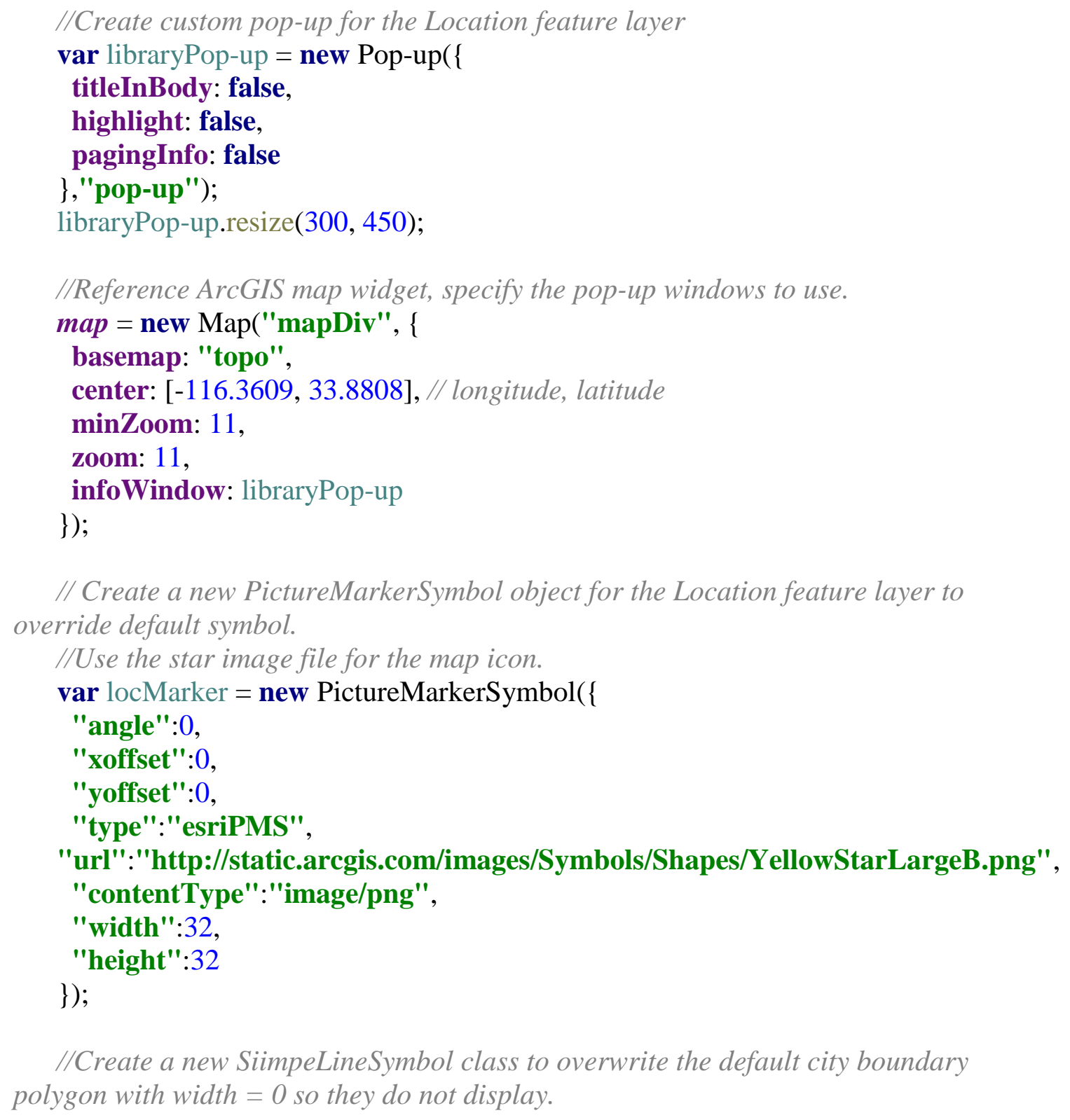


var jurisdictionBoundary = new SimpleLineSymbol $(\{$

type: "esriSLS",

width: 0 ,

style: "esriSLSDot"

\}$)$;

//Apply the symbol definitions to the respective layers using the SimpleRenderer class

var locRenderer $=$ new SimpleRenderer(locMarker);

var boundaryRenderer $=$ new SimpleRenderer(jurisdictionBoundary);

//Create pop-up templates for locations, vendors, and permit data feature layers

var locTemplate $=$ new Pop-upTemplate $(\{$

title: "(Click arrow for permit information)",

description: " $\langle\mathrm{b}\rangle\{$ LocTitle $\}</ \mathrm{b}\rangle\langle\mathrm{br}\rangle\langle\mathrm{br}\rangle$ Click image for more pictures" +

" $<$ a href $=\{$ PhotoURL $\}$ target $=$ '_blank' $><$ img src $=\{$ Photo_TH $\}$ alt $=\{$ LocTitle $\}$

height=188 width $=250\rangle\langle/ \mathrm{a}\rangle\langle\mathrm{br}\rangle "$

" $\langle\mathrm{b}\rangle$ Site Offered By: $</ \mathrm{b}\rangle\langle$ br $\rangle\{$ OffByType $\},\{$ OffByComp $\}\langle$ br $\rangle\langle$ br $\rangle "+$

" $<$ b $>$ Location Size: $</ \mathrm{b}>\{$ LocSize $\}<$ br $>"+$

" $<\mathrm{b}>$ Propery Size: $</ \mathrm{b}>\{$ PropSize $\}<\mathrm{br}>"+$

" $\langle$ b $>$ Number of Bedrooms: $\langle/$ b $>\{$ NumBeds $\}\langle$ br $\rangle\langle$ br $\rangle "+$

" $<\mathrm{b}>$ Production Hours: $</ \mathrm{b}>$ \{ProdHours\} $\langle\mathrm{br}\rangle "+$

" $<$ b $>$ Max. Number Vehicles: $</$ b $>\{$ MaxNumVeh $\}<$ br $>$ " +

" $<$ b $>$ Max. Number Persons: $</$ b $>\{$ MaxNumPersons $\}<$ br $>$ " +

" $\langle$ b $>$ Photography Only: $\langle/$ b $>\{$ PhotosOnly $\}<$ br $\rangle\langle$ br $\rangle "+$

$/ / "\langle b\rangle$ Contact: $\langle/ b\rangle\langle b r\rangle\{$ relationships/3/Person $\}\langle b r\rangle\langle b r\rangle "+$

" $\langle\mathrm{b}\rangle$ Address: $\langle/ \mathrm{b}\rangle\langle\mathrm{br}\rangle$ Address $\}\langle\mathrm{br}\rangle\{$ City $\}\langle\mathrm{br}\rangle\langle\mathrm{br}\rangle "+$

" $\langle$ b $>$ Category: $\langle/ \mathrm{b}\rangle\langle$ br $\rangle\{$ LocCat $\}\langle$ br $\rangle "+$

" $\langle$ b $>$ Type: $\langle/$ b $\rangle\langle$ br $\rangle\{$ LocType $\}\langle$ br $\rangle\langle$ br $\rangle$ " +

" $\langle$ b $>$ Primary Style: $\langle/ \mathrm{b}\rangle\langle$ br $>\{$ LocArchStyle $\}<$ br $\rangle "+$

" $\langle$ b $\rangle$ Secondary Style: $\langle/ \mathrm{b}\rangle\langle$ br $\rangle\{$ LocOtherArch $\}\langle$ br $\rangle\langle$ br $\rangle "+$

" $\langle$ b $>$ Summary: $\langle/$ b $\rangle$ LocShortSum $\}<$ br $\rangle\langle$ br $\rangle "+$

" $<$ b $>$ Description: $</$ b $>\{$ LocLongSum $\}<$ br $><$ br $>$ " +

"<b>Notes: $</ \mathrm{b}>$ SPecialComments $\}<\mathrm{br}>$ "

\}$)$;

var vendorTemplate $=$ new Pop-upTemplate $(\{$

title: "Business Information",

description: "<b $>$ Category: $\langle/$ b $\rangle$ Category $\}<$ br $\rangle\langle$ br $\rangle$ " +

" $\langle\mathrm{b}\rangle$ Name $</ \mathrm{b}\rangle\langle$ br $\rangle "+$

" $\{$ Name $\}<$ br $\rangle\langle$ br $>$ " +

" $\langle\mathrm{b}\rangle$ Address $\langle/ \mathrm{b}\rangle\langle\mathrm{br}\rangle$ " +

" $\{$ Address $\}<$ br $>$ " +

" $\{$ City $\},\{$ State $\}\{$ ZipCode $\}<$ br $>\langle$ br $>"+$

" $\langle\mathrm{b}\rangle$ Phone $</ \mathrm{b}\rangle\langle\mathrm{br}\rangle$ " +

" $\{$ VenPhone $\} "$ 


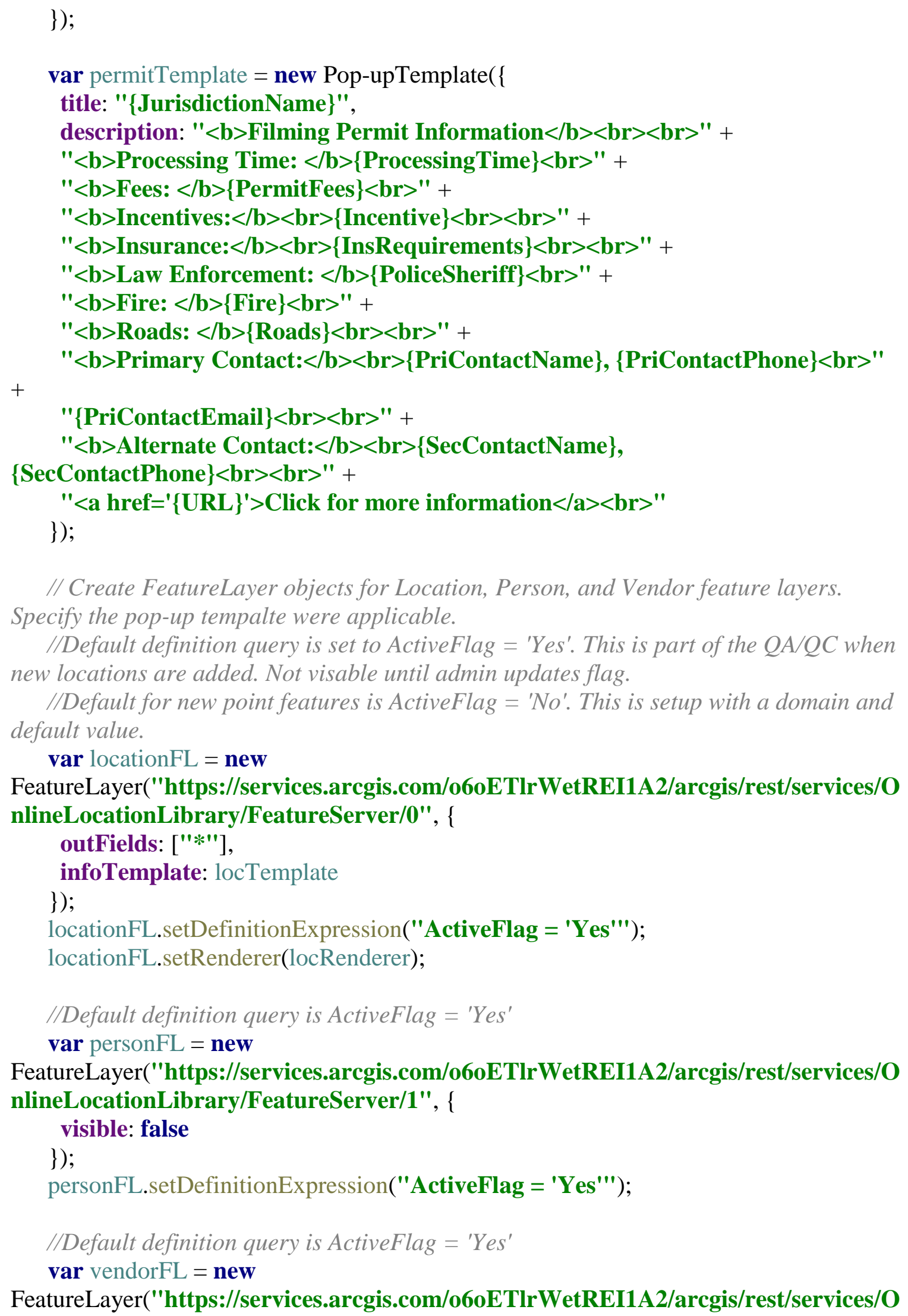




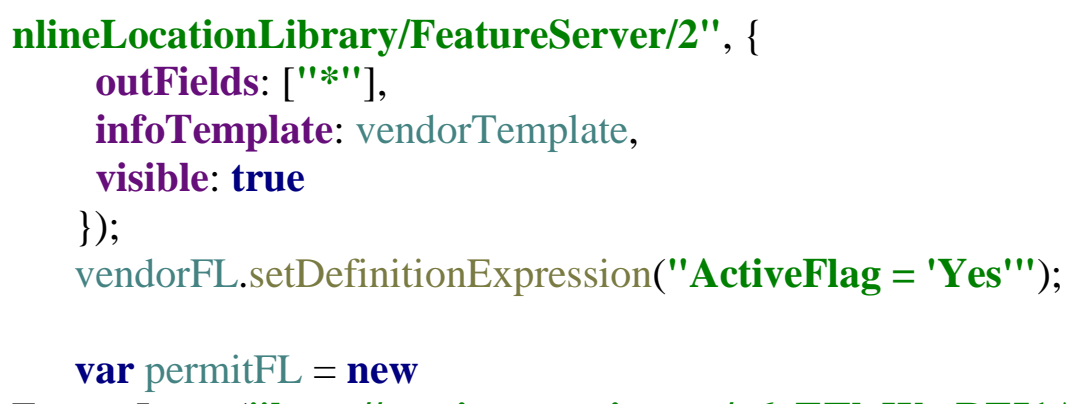

FeatureLayer("http://services.arcgis.com/o6oETlrWetREI1A2/arcgis/rest/services/O nlineLocationLibrary/FeatureServer/3", \{ outFields: ["*"], infoTemplate: permitTemplate

);

permitFL.setRenderer(boundaryRenderer);

// Add all layers to the map object.

map.addLayers([permitFL, vendorFL, personFL, locationFL]);

//Create the structure for the layout of the application including the accordion pane on the left.

//Base is a BorderContainer. Header and Center panes are ContentPane classes.

//AccordionContainer on the left is added later since feature attributes must be retrieved to create definition

// query for filter functions and container titles.

//The following code written as part of creating the location filter feature in the Accordion pane is original code

//by Mark E. Gilbert.

var mainContainer = new BorderContainer (\{design: "headline" $\}$, "appLayout" ); var headerPane = new ContentPane(\{region: "top" \}, "topPane"); mainContainer.addChild(headerPane); var centralPane = new ContentPane( \{region: "center" \}, "centerPane"); mainContainer.addChild(centralPane);

//Wait for the first layer to be added to the map before building the BorderContainer //otherwise the setDefinitionExpression statements will cause errors.

map.on("load", function() \{

//Initialize query task to create the cross reference table between location category integer values and text descriptions.

//Integer values are used for the definition queries and text descriptions are used for the ContentPane titles.

var catCrossRefQueryTask $=$ new QueryTask("http://services.arcgis.com/o6oETlrWetREI1A2/arcgis/rest/services/Onli neLocationLibrary/FeatureServer/7"); 
//Query object for the location query

var locQueryTask = new

QueryTask("'https://services.arcgis.com/o6oETlrWetREI1A2/arcgis/rest/services/Onl ineLocationLibrary/FeatureServer/0");

//This is the query of the LocatoinCategory table to build the cross reference table to translate values into text.

//Category is the integer value used for the definition query and CatSubTypes is the text description for titles.

var catCrossRefQuery = new Query();

var categoryCrossRef $=\{\}$;

catCrossRefQuery. where = "Category $<>$ "'"; //Return all rows

catCrossRefQuery.returnGeometry = false;

catCrossRefQuery.outFields = ["Category", "CatSubtypes"];

catCrossRefQueryTask.execute(catCrossRefQuery, processCrossRef);

//Process the results to create a dictionary with the integer value as key and text description as value. Used when creating the accordion panes.

function processCrossRef(results) \{

var resultCount $=$ results.features.length;

for $(\operatorname{var} i=0 ; i<$ resultCount; $i++)\{$

categoryCrossRef[results.features[i].attributes.Category] =

results.features[i].attributes.CatSubtypes;

\}

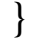

//Create the All Locations accordion pane the removes the definition query from Locations to display all locations.

//When a particular accordion pane is selected and the definition expression is applied, the Search widget

//only displays results from the features visible based on the definition expression and not all features.

leftPane = new AccordionContainer $(\{$ region: "left", splitter: false $\}$, "leftCol" $)$;

leftPane.addChild(new ContentPane( \{

id: 999 ,

title: "<b $>$ All Locations $</ \mathrm{b}>$ ",

content: "Clear filter and display all locations."

\}));

//Query the location feature layer for all locations

//All locations will be iterated over to get their location categories used for building the filter panes.

var locQuery = new Query ();

locQuery. where = "ActiveFlag = 'Yes"'; //Return all active rows for inclusion in feature filter 
locQuery.returnGeometry = true;

locQuery.outFields = ["ObjectID", "LocCat", "LocTitle"];

locQueryTask.execute(locQuery, processCategory);

//Processing the results of the location query to create the content panes, setup the definition expression, and

/ladd the location titles as content to their respective content panes.

function processCategory (results) \{

// console.log("Inside processCategory()");

// console.log(results.features)

// console. $\log ($ categoryCrossRef);

var resultCount $=$ results.features.length;

for $(\operatorname{var} \mathrm{i}=0$; $\mathrm{i}<$ resultCount; $\mathrm{i}++)\{$

var featureAttributes = results.features[i].attributes;

var filterCategory = categoryCrossRef[featureAttributes.LocCat]; //Use LocCat integer as an index to translate it to description of category

var catTitle = featureAttributes.LocTitle; //Use LocTitle for the content in the pane.

var locOBID = featureAttributes.OBJECTID; //Use the ObjectID for showing the рор-ир

// console.log("filterCategory: " + filterCategory, "catTitle: " + catTitle );

// Checking to see if the content pane already exists. If there are multiple locations with the same

// LocCat we do not want to try and create a new content pane. Only need each LocCat once.

//Setting up content panes to support hyperlinked location titles that display the pop-up when clicked.

if (document.getElementById(featureAttributes.LocCat) === null) \{

leftPane .addChild(new ContentPane( \{

id: featureAttributes.LocCat,

title: "<b $>$ " + filterCategory + "</b $>$ ",

content: "<a href='\#' onclick='displayPop-up(" + locOBID + ")'>" + catTitle $+"</ \mathbf{a}>"$

\}$))$

\} else \{

var curContent $=$

document.getElementById((featureAttributes.LocCat)).innerHTML;

document.getElementById((featureAttributes.LocCat $))$.innerHTML = curContent + "<br $>$ " + "<a href='\#" onclick='displayPop-up $("+\text { locOBID + " })^{\prime}>$ " + catTitle + "</a $>$ ";

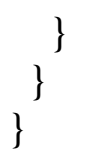


//Put is all together.

mainContainer.addChild(leftPane);

mainContainer.startup();

//This is watch event updates the definition expression on the Location feature layer to filter the map based on the user selection.

//All Locations was created with an ID of 999. All others were given the ID equal to the location category integer value.

//onShow seems to fire before the child is selected which results in the previously selected ID displaying.

//Using the watch reports the correct child widget.

leftPane.watch("selectedChildWidget", function(name, oVal, nVal)\{

// console.log("Name: " + name + "Selected child changed from ", oVal.id, " to ", nVal.id);

var catFilter;

if $(\mathrm{nVal} . \mathbf{i d}==999)\{$

catFilter $=$ "ActiveFlag $=$ 'Yes"'; //if the All Locations pane is selected, set

definition expression to all locations

\} else \{

catFilter $=$ "LocCat $=$ "' + nVal.id + "' AND ActiveFlag = 'Yes" ";

\}

locationFL.setDefinitionExpression(catFilter);

// console.log(locationFL.getDefinitionExpression());

\}$)$

\}$)$

//Add the home button widget to the map

var homeButton = new HomeButton $(\{$

map: map

\}, "HomeButton");

homeButton.startup();

// Add the basemap toggle widget to the map

var baseMapToggle = new BasemapToggle $(\{$

map: map,

basemap: "satellite"

\}, "baseMapToggle");

baseMapToggle.startup();

//Create the search function on the Location Feature Layer

//An empty array is assigned to the sources property to purge the default World

Geocoding Service

var searchFL = new Search $(\{$

map: map,

sources: [],

zoomScale: 10000 


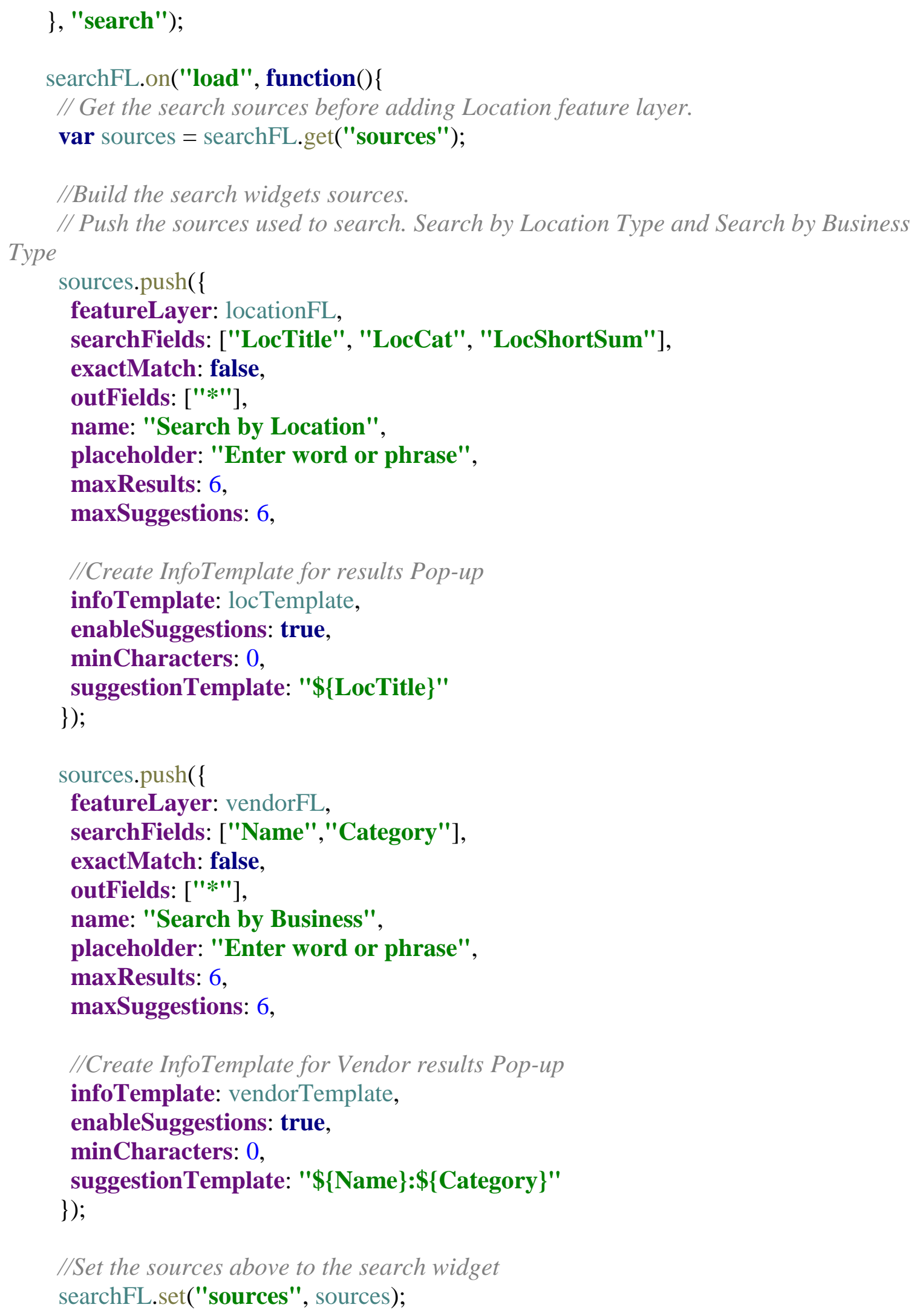


\});

searchFL.startup();

\}); 


\section{Appendix C. CSS Source Code}

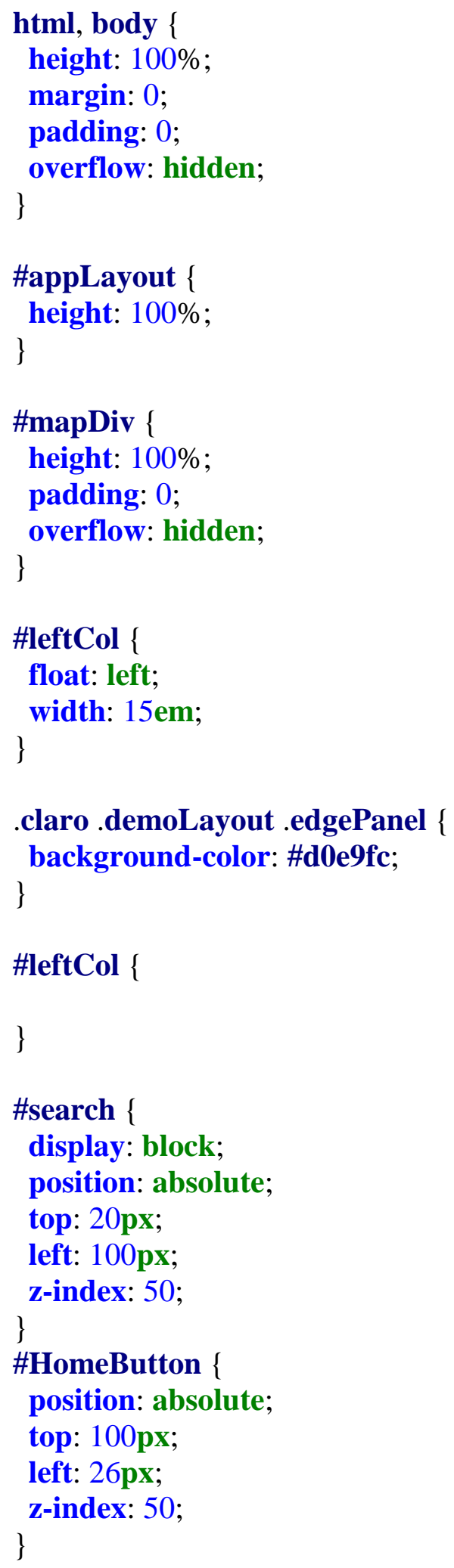




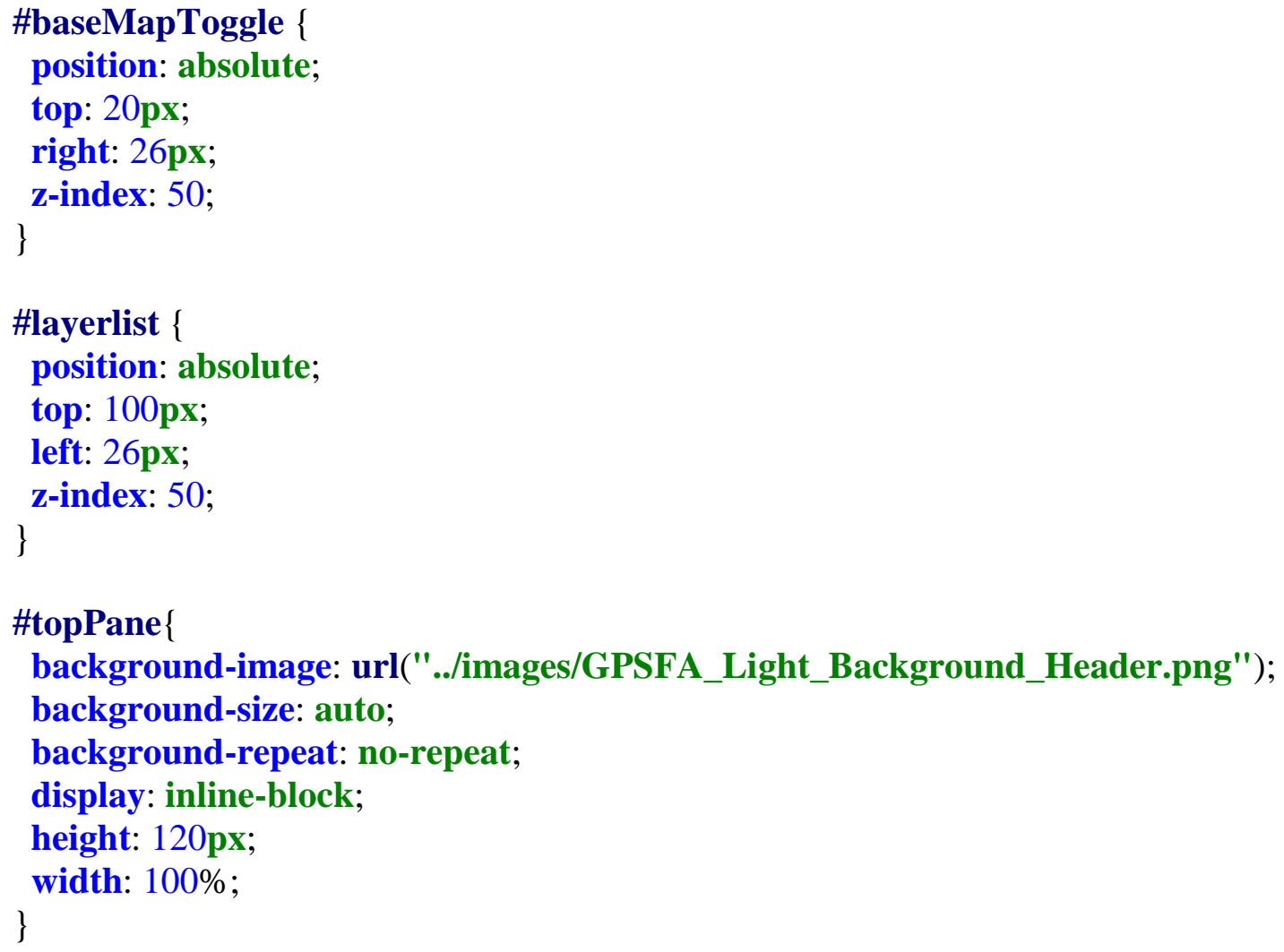




\section{Appendix D. Python Script}

\#Created by Mark E Gilbert

\#August 2016

\#This script reads CSV files from a location and iterates through the files. \#For each file, it takes the filename, excluding the extension, and creates a \#file geodatabase domain using the two fields in the file. The file name is used \#for the domain name. Fields in the file need to be the domain coded value followed \#by the text description separated by a comma. The files geodatabase needs to \#exist as does the Location feature class with the subtypes defined.

\#Field LocType also needs to be present in the feature class.

\# Syntax

\# CreateDomain_management (in_workspace, domain_name, \{domain_description\}, \{field_type $\},\{$ domain_type $\},\{$ split_policy $\},\{$ merge_policy $\}$ )

\# TableToDomain_management (in_table, code_field, description_field, in_workspace, domain_name, \{domain_description $\},$ update_option $\}$ )

\# arcpy.CreateDomain_management(inputWorkspace, "Agriculture", "Agriculture category", "TEXT", "CODED")

\# arcpy.AssignDomainToField_management (in_table, field_name,domain_name, \{subtype_code $\}$ )

\# execfile(r'C:IUsers\mgilbert\Documents\Film

AlliancelFrom_LeviVincent\Tabular\NoSpatialReferencelcreateDomains.py')

\#Import modules

import arcpy

import os

\#set the enviornment in which to work.

arcpy.env.workspace $=$ "C: $\backslash$ Users $\backslash$ mgilbert $\backslash$ Documents $\backslash$ Scratch"

\#\#arcpy.env.workspace = "C: \Users\mgilbert\Documents $\backslash$ Film Alliance"

\#set the file geodatabase in which to work.

inputWorkspace $=$ "ImportTesting.gdb"

\#\#inputWorkspace = "GPSFALocationLibrary.gdb"

\#define the folder that contains the delimited files for each category

domain_folder = "C: Users\mgilbert\Documents\Film

AlliancelFrom_LeviVincent|TabularlNoSpatialReferencelDomains"

\#define the feature class in the workspace to receive the domains. In this case, it's the Location FC. Define the FC attribute

\#that will get the domain assigned to it. In this case, LocType in the Location FC.

$\mathrm{fc}=$ arcpy.env.workspace + "\I" + inputWorkspace + "\\Location" 
fieldName = "LocType"

\#get the list of delimited files from the subdirectory

domainFiles $=$ os.listdir(domain_folder)

print("Domain load started...")

\#interate through all the delimited files.

for file in domainFiles:

\#\# print file

domainTitle = file.split(".")[0] \#use the file name (without extenstion) as the name of the domain

\#\# print domainTitle

\#arcpy tool for converting a table (the delimited file data) and making it into a domain.

if domainTitle not in("Architecture", "Offered By Type", "General", "YesNo",

"VendorCategory"): \#These domains should be managed manually

arcpy.TableToDomain_management (domain_folder + "II" + file, domainTitle,

domainTitle + " category", inputWorkspace, domainTitle)

print("Domain load comleted.")

\#get the list of subtypes from the Location FC. Function returns a dictionary. These will be used to create a dictionary for assigning

\#the correct domain.

\#\#\#Invert dictionary adapted from Stack Overflow

http://stackoverflow.com/questions/1031851/python-best-way-to-exchange-keys-withvalues-in-a-dictionary\#\#\#

subTypesDict $=$ arcpy.da.ListSubtypes $(\mathrm{fc})$

invertedSubTypeDict $=\operatorname{dict}([(\operatorname{str}(\mathrm{v}[" N a m e "]), \mathrm{k})$ for $\mathrm{k}, \mathrm{v}$ in subTypesDict.items() ]) \# Invert to look up code from value

print("Domain assignment started...")

for file in domainFiles:

domainTitle = file.split(".")[0]

if domainTitle not in("Architecture", "Offered By Type", "General", "YesNo",

"VendorCategory"): \#These domains should be managed manually arcpy.AssignDomainToField_management (fc, fieldName, domainTitle, invertedSubTypeDict[domainTitle])

print("Domain assignment by SubType comleted.") 